\title{
THE ENVIRONMENTS OF OBSCURED QUASARS
}

\author{
Kristen M. Jones \\ Charlottesville, VA, USA \\ M.S. of Astronomy, University of Virginia, 2011 \\ A Dissertation Presented to the Graduate \\ Faculty of the University of Virginia \\ in Candidacy for the Degree of \\ Doctor of Philosophy \\ Department of Astronomy \\ University of Virginia \\ July 2016
}

B.S. of Physics and Astrophysics, University of Wisconsin-Madison, 2009

Committee Members:

Dr. Mark D. Lacy

Prof. Mark D. Whittle

Prof. Aaron S. Evans

Prof. Dinko Pocanic 
(C) Copyright by

Kristen M. Jones

All rights reserved 


\begin{abstract}
The study of Active Galactic Nuclei (AGN) hinges on observations that probe the underlying characteristics of the supermassive black hole and the host galaxy in which it is found. Traditional identification of an AGN has relied on broad emission lines and high uv/optical luminosities. In the past fifteen years, infrared (IR) observations have revealed an AGN population that is shrouded in dust and demonstrated that such a population makes up a significant (at least 50\%) contribution of the total AGN population. It is therefore vital that future work on all types of AGN be designed to include both obscured and normal or unobscured objects in their samples.

With this in mind, this dissertation explores the environments associated with dust-obscured luminous objects, particularly Luminous Infrared Galaxies (LIRGs) and Type 1 and Type 2 quasars. The environment at all scales (from the nuclear $(<1 \mathrm{pc})$ to the halo $(10 \mathrm{~s}$ to $100 \mathrm{~s}$ of $\mathrm{Mpc}))$ plays a role not only in creating AGN but in how they and their host galaxies evolve with time to become (or be observed as) vastly different populations. This makes it a challenging field to explore, but one with many avenues open to investigation.

First, I reveal the complexities involved in the 1-2 kpc (circumnuclear) environment scale of LIRGs, objects where AGN and star formation processes can both play an equivalent role. Mid-infrared high resolution images reveal diverse morphology that suggests the importance of star formation in this scale in powering LIRG luminosity and channeling gas into the nuclear $(<100 \mathrm{pc})$ region. I identify a circumnuclear spiral within an elliptical galaxy, as well as possible disky structures in another two objects. The final two objects appear to be unresolved. I find the circumnuclear region emits a dominant proportion of the total LIRG luminosity for the majority of the objects in the sample, with minimum surface brightness densities on order
\end{abstract}


of $10^{10} L_{\odot} k p c^{-2}$, approaching the surface brightness densities found in star forming regions in other galaxies.

Secondly, I investigate the halo-scale clustering of unobscured and obscured quasars in order to explore the environments in which these luminous, AGN-dominated sources form. With the difficulty of identifying and detecting the dust-shrouded obscured quasars, the large-scale environment of the population has been relatively understudied in the past decade, but could hold the key to distinguishing between whether the obscuration is a temporary stage resulting from a recent major merger (the evolutionary model), or a permanent feature of the dust structures of the host galaxy (the orientation model). The latter predicts that the environments in which both types of quasars are found should be similar, and indeed in the range of $1.3<z<2.5$ for Spitzer IR-selected obscured and unobscured quasars, I calculate similar median clustering amplitudes, indicating nearly identical large-scale environments. These results are discussed in the context of numerous other findings in the field. 


\section{Acknowledgements}

This dissertation would not have been possible without the extensive support, compassion, and contributions of many, many people in my life. I am grateful to each and every one of my friends, family, and colleagues.

First I must thank my advisor, Mark Lacy, whose patience, passion for science, and belief in me and my work has been the cornerstone of my graduate career and my decision to continue pursuing science. In addition, I am forever grateful to the other members of my dissertation committee, who have helped me hammer the distributed components of my research into a sword (I intend to use it going forward).

I would not be here without the groundwork that my family has laid for me, creating a place from which I could evolve. To Stefanie: $<3$ thank you for everything. To Mom: thank you for always believing in me. To Jessica and Shawn: thank you for the joy and kindness you offer me, and the home you have opened for me every time I visit you. To Ryan: you always help me remember that persistence and hard work are the key components in life. To Michaela: few astronomers are lucky enough to find new luminous things that are wonderous beyond explanation-I'm fortunate that I found one in you. Robin: thank you for being here for me for so very long Louise, Patrick (and new wee Patrick): words cannot express what you have given me, so I will give you hugs instead. To Dad: I wish you could be here, but I know you'd be proud.

My career so far has given me the opportunity to work with or for some of the most kind, generous, dedicated, and intelligent scientists I have ever met. I am grateful for the members of my undergraduate reserach lab (Peter Timbie, Amanda Gault, Siddharth Malu, Peter Hyland, Emily Barrentine, Allison Noble, and Melissa Lucero) who taught my how to begin to think and how to have fun (and for introducing me to 
Firefly). I am grateful for those advisors who sponsored me in various research roles and taught me to learn from new perspectives (S. Tom Megeath, Mary Lou Roberts, Ganesh Rajagopalan, Dana Whitlow, and Rich Bradley). I am grateful to Margrethe Wold for giving me the opportunity to work on new data in Denmark for 6 months, and simultaneously providing my first opportunity to explore the world-I will never forget the way this has opened my eyes. I am grateful to the Dark Cosmology Centre and every person there employed during my stay, who greeted me with kindness and warmth and understanding and brought the spark of joy back into my research career (especially Daniele Malesani, Radek Wojtak, Ia Kochiashvili, Martina Falco, Johannes Zabl, Ardis Eliasdottir, Julie Meier, Michelle Cumming Lokkegard, Thomas Greve, Bitten Gullberg, Matteo Cataneo, Stefan Geier, Teddy Frederikson, Ece Eser, and Bo Milvang-Jensen). Finally, I would not be the scientist I am today without my passion for righting injustice in academia, for which I must thank Melissa Kyle, Maya Holtzmann, Jeff Shokler, Katie Zaman, Jackie Vinson, Nick Murphy, Jedidah Isler, Anup Gampa, and Chanda Prescod-Weinstein.

I was fortunate to have so many people in my life reach out to mentor me as a scientist. I would like to especially thank Peter Timbie, Susan Nossal, Jeff Shokler, Maya Holtzmann, Bonnie Valant-Spaight, Dan McCammon, Lisa Everett, Mike Winokur, Lawrie Balfour, Chris Salter, Tapasi Ghosh, Robert and Helen Minchin, Ed Murphy, Phil Arras, Allison Peck, and Anne Verbiscer.

To Vincenza, Nicole, Monique, Sara, Victoria, Heather, Allison, Paddy, Sarah, Pallavi, Loreto, Hibbard, Emily, Sandy, Lauren, Katy, Amanda, Trey, Rachael, Bill, Anya, Tuck, Nick, Tim, Chris, Tang, Abel, Charles, Paul, Brian, Guillermo, Kim, Jake (both redbeard and henry), Joanna, Genevieve, Joleen, Gail, Nick, Rachel, Jackie, all other members of the University of Virginia Astronomy Department and 
the National Radio Astronomy Observatory, and all my other friends and colleagues who have stood by me throughout this long experience: thank you a thousand times. 


\section{Table of contents}

$\begin{array}{ll}\text { Abstract } & \text { ii }\end{array}$

Acknowledgements iv

1 Introduction to Supermassive Black Holes, Galaxy Formation and Evolution, and Our Modern Understanding of Quasars 1

1.1 Why Study Quasars? (Quasars as a Means to Studying Supermassive Black Holes) . . . . . . . . . . . . . . . . . . . . . . 1

1.2 Intrinsic Quasar Characteristics (The Overall Relation between Quasars and AGN) . . . . . . . . . . . . . . . . . . 3

1.3 The Role of Environment (The Quasar-Galaxy Evolution Relationship) 7

1.4 The Complexity of AGN (Multiple Levels of Understanding Quasars) 10

1.5 Questions Underlaying This Dissertation . . . . . . . . . . 13

2 High Resolution Mid-Infrared Imaging of the Active Regions in Five $\begin{array}{lr}\text { Luminous Infrared Galaxies } & 14\end{array}$

2.1 Abstract . . . . . . . . . . . . . . . . . . . . . . . . . . . . . . . . . .

2.2 Introduction . . . . . . . . . . . . . . . . . . . . 15

2.3 Sample, Observations, and Data Reduction . . . . . . . . . . . 16

2.3.1 The Sample . . . . . . . . . . . . . . . . 16

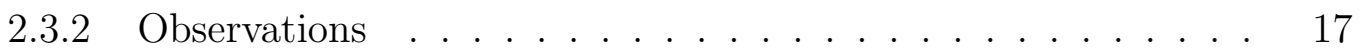

2.3.3 Data Reduction . . . . . . . . . . . . . . . . 18

2.4 Results . . . . . . . . . . . . . . . . . . . . . . . . . . . . . . . . . . .

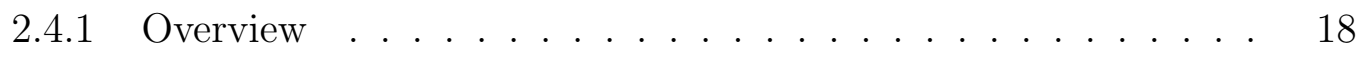

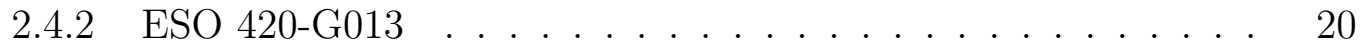

2.4 .3 NGC $2369 \ldots \ldots \ldots . \ldots \ldots$

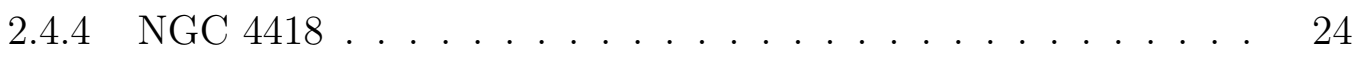

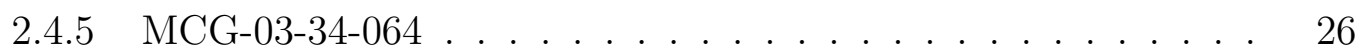

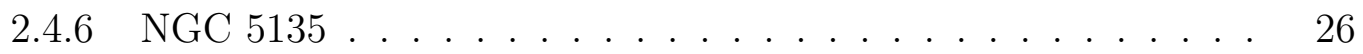

2.5 Discussion . . . . . . . . . . . . . . . . . . . . . . 29

2.5.1 Diversity of Nuclear Morphology . . . . . . . . . . . . . . . 29

2.5.2 Brightness Surface Density . . . . . . . . . . . . . . . 31 
2.5.3 The Nature of the Circumnuclear Spiral found in ESO 420-G013 34

2.6 Conclusions . . . . . . . . . . . . . . . . . . . 37

2.7 ACKNOWLEDGEMENTS . . . . . . . . . . . . . . . . 38

3 Clustering in SERVS at High-Redshift: No Difference in Halo-Scale Environments between Obscured and Unobscured Quasars $\quad 40$

3.1 Abstract . . . . . . . . . . . . . . . 40

3.2 Introduction . . . . . . . . . . . . . . . . . . . . 41

3.3 Sample Selection . . . . . . . . . . . . . . . . . 45

3.3.1 Quasar Selection and Obscured Quasar Identification . . . . . 45

3.3.2 Galaxy Selection and Quasar Association Determination . . . 46

3.4 Measuring Environmental Richness . . . . . . . . . . . . . . . 47

3.4.1 Cross-correlation Functions . . . . . . . . . . . . . . 47

3.5 Results . . . . . . . . . . . . . . . . . 51

3.5.1 Background Galaxy Counts . . . . . . . . . . . . 51

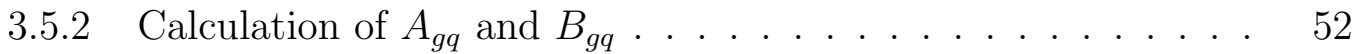

3.5.3 Results as a Function of Redshift and Type . . . . . . . . . . 55

3.6 Conclusions . . . . . . . . . . . . . . . . . . 58

3.6.1 Comparison to Other Work in the Literature . . . . . . . . 58

3.6.2 Overall Findings . . . . . . . . . . . . . . . . 60

4 Conclusions and Future Work $\quad 62$

4.1 Overall Conclusions of This Dissertation . . . . . . . . . . . . . . 62

4.1.1 Halo-scale Environments of Obscured and Normal Quasars at

High Z . . . . . . . . . . . . . . . . . . . . . . . . . . . . . . . 62

4.1.2 Circumnuclear Environments and the Presence of AGN in LIRGs 64

4.2 Future Work . . . . . . . . . . . . . . 66

4.2.1 Approaching a True Understanding of AGN and the Role of Sample Selection . . . . . . . . . . . 66

$\begin{array}{ll}\text { Appendices: Future Proposals } & 68\end{array}$ 


\section{Chapter 1}

\section{Introduction to Supermassive}

\section{Black Holes, Galaxy Formation}

and Evolution, and Our Modern

Understanding of Quasars

\subsection{Why Study Quasars? (Quasars as a Means to Studying Supermassive Black Holes)}

Quasars are unique beacons in the vast darkness of the cosmos. Their brilliance spans the baryonic eras of cosmic history, and stems not from gleaming stars but paradoxically from one of the most mysterious and bizarre entities in the universe - supermassive black holes, also known as SMBHs. At first glance, one would not expect a black hole to be luminous, much less bright enough to be visible at redshifts of $\mathrm{z} \sim 7$. However, the interaction between a SMBH and the material found in its 
neighborhood provides this powerful luminance, and reveals knowledge that pushes at the boundaries of our understanding of both physics and astronomy. SMBHs are where Einstein's Relativity and Newtonian Gravity manifestly demonstrate their distinctions. These beasts have masses of $10^{6} M_{\odot}$ or more, and how they are formed, and how they influence, and are influenced by, their environment, are potent matters for debate. Quasars are the quintessential astronomical curiosity. Detected in early radio surveys in the 1950s as radio sources with no obvious optical counterpart, they became known as quasi-stellar radio sources in the early sixties when the first associated optical observations detected faint blue stellar-like optical emission. The spectra of the associated "stars" defied explanation, revealing strong spectral emission lines not associated with any known element at the time. At last a solution consistent with the unusual data that they furnished was revealed by Schmidt (1963) and Bolton et al. (1965): their spectral information was shown to be consistent with known elements (particularly hydrogen) but at huge redshifts, far beyond anything known at that time. At first this was a difficult pill for astronomers to swallow, as the high redshifts required the source to be extremely luminous in order to be observed today. Scientific minds thus turned to understanding the source of the extreme luminosities and unusual spectral shape that were being revealed. Eventually the interpretation has settled to a scenario in which a disk of material rotates around a mass so huge, and on a size scale so small that it would have to be a black hole more massive than any previously postulated (e.g.,Lynden-Bell (1969))

To study the environments in which such SMBHs form, it is therefore natural to pinpoint the most obvious SMBH-dominated systems, namely quasars (also called QSOs (Quasi-Stellar Objects). These objects have such high luminosities that models suggest it is extremely unlikely for their power source to be anything other than a 
SMBH. This luminosity allows quasars to be observed over a wide range in redshifts - the most distant quasar that we currently know has $z \sim 7$ (Mortlock et al. (2011)). Quasars appear to occupy one small part of an evolutionary sequence of galaxies (e.g., Hopkins et al. (2008), Sanders et al. (1988c)), and are thought to be preceded by a major merger event that drives large quantities of dust and gas into the circumnuclear region, where processes feed this "fuel" further onto a central accretion disk and thence into the black hole that it surrounds (e.g., Sanders (1998), Croft et al. (2006), Bournaud et al. (2010), Netzer \& Marziani (2010), and Silk \& Nusser (2010)) and feedback between the quasar and the circumnuclear and nuclear regions can in turn blow the fuel out of the region (e.g. Schawinski et al. (2006)). While quasars are not common in the local universe, they may be analogous to the other types of active galactic nuclei (AGN) that have been observed today, such as Seyfert galaxies, LINERs, and Ultra Luminous Infrared Galaxies (Sanders \& Mirabel (1996), Silk

\& Rees (1998)). As the high luminosity end of this AGN sequence, and hence as objects visible over a wide redshift range, quasars are ideal candidates for exploring the environments of active SMBH throughout the history of the universe (e.g., Ho $(2004))$.

\subsection{Intrinsic Quasar Characteristics (The Overall Relation between Quasars and AGN)}

One of the things that time has revealed is that supermassive black holes are not exclusive to currently active nuclei. In fact, they appear to be present in the central region of nearly every massive galaxy (Kormendy (2001), Schödel et al. (2002), Atkinson et al. (2005), Pastorini et al. (2007), Reines et al. (2014)), including our 
own Milky Way. Research has also found them within ntermediate mass galaxies and dwarf galaxies (Reines et al. (2011),Greene (2012), Reines et al. (2013), Seth et al. (2014), Reines (2014)), particularly for galaxies that contain any spheroidal or bulge-like component. SMBHs are thus theoretically as intrinsic a part of a galaxy as gas, dust, dark matter, and stars. There is, however, a separation between those nuclei which contain quiescent SMBH and those that are "active" - e.g., those that are emitting in some fashion. In practice, the standard criterion that defines an active galactic nucleus is that it is accreting at $\geq 0.1 \%$ of the Eddington limit. (An aside: The Eddington limit is defined as the luminosity at which the force from the radiation pressure (created by interactions between particles and photons in the region, such as electron scattering) equals the gravitational force of the object under consideration. At this point, no further accretion is possible. The Eddington rate is the term used for the accretion rate at which a black hole emits at the Eddington luminosity. This is often used interchangeably with the term radiative efficiency, which is defined as the ratio between the black hole's luminosity and the energy provided by the gas accretion rate $\dot{M}$ (the potential energy is from accretion at a certain rate is $\dot{M} c^{2}$ ). An efficiently radiating $\mathrm{SMBH}$ is thus one that can produce a luminosity close to the Eddington limit).

The source of luminosity from an accreting SMBH is multifold. Firstly, "frictional" (dissipative) heating of the material in the rotating accretion disk, quite close to the accretion radius of the SMBH, produces a high-temperature-peaked blackbody spectrum that is reprocessed in part into a complex spectral shape by the dust, gas, and (occasionally) molecular clouds that surround the nuclear and circumnuclear regions. Secondly, a collimated outflow (or jet) that originates from the SMBH itself (through processes that are not yet fully understood) serves as the source of radio 
continuum emission (generated through the synchrotron process), and may contribute to further spectral lines as it drives shocks in the nuclear and circumnuclear regions. Thirdly, a hot corona of gas around the accretion disk that can up-scatter background photons through the Inverse-Compton process creates x-ray radiation that approximates a power law. An AGN may also produce cosmic rays and high energy particles, though the exact means of production is currently unclear. Some AGN have a soft $\mathrm{x}$-ray continuum component in addition to the x-ray power-law component; the origin of this soft x-ray emission is also unknown. AGN are often identifiable by their broad spectral energy distribution (SED) that results from a combination of these different processes. They are also identifiable from distinctive emission lines, often demonstrating rapid rotation via Doppler broadening that results from proximity to the SMBH.

Of course each individual AGN is a more complex system. Some are radio-quiet, some have insignificant x-ray luminosity, some have diminished optical emission, or optical and NIR emission lines obscured by excess dust, while others have nearly all their AGN contributions to their SED outshone by the occurence of high star formation rates (especially a problem for unresolved extragalactic sources). Further, since the SMBH and its immediate surroundings are of very small size $(<1 \mathrm{pc})$, the observed SED can vary on the order of weeks to years. Finally, the accretion rate and efficiency of each AGN can vary, not just between types but on short timescales.

Overall, the observed characteristics of AGN are not only different due to different combinations of the individual components of the nucleus or different accretion efficiencies: they also are contaminated by the presence of the host galaxy in which the AGN exists, as well as any unique characteristics of that host galaxy's environment. This makes their study - the study of AGN properties as a function of time, redshift, 
host-galaxy evolution or characteristics, luminosity, or SMBH mass - particularly challenging. As a result, most work in the field has developed around defining sample populations of AGN - groups of objects that, due to some observational property, can be assumed to represent the same intrinsic population - for further study. The relevant observational property can focus on direct observables such as flux density in the $24 \mu \mathrm{m}$ band; the strength of the $11.3 \mu \mathrm{m}$ PAH line; the presence of certain emission lines such as $\mathrm{H}-\alpha$ or $\mathrm{H}-\beta$ of a particular width; or ratios of emission line fluxes or equivalent widths such as those used in BPT diagrams (Baldwin et al. (1981)). But observational properties for distinguishing a distinct population can also focus on more indirect properties that require assumptions or understanding from experimental relations, such as the bolometric luminosity derived from IR flux; star formation rates calculated using the Kennicutt relations; calculating the slope of radio emission to determine the dominant processes in a region; or mass derived from the $M_{B H}-\sigma_{*}$ relation). Regardless, for each study the aim is to find a suitable quantity through which to identify an intrinsic property of an AGN.

The definition of quasars or QSOs as a population of interest relies on both luminosity and redshift as observable properties. Originally, they were distinguished by outshining the rest of their host galaxy in the optical regime (leading to the quasistellar part of their names). Secondly, they are found at higher redshifts, $\mathrm{z}>0.1$. Finally, their bolometric luminosity is not only significantly greater than their host galaxies, but also exceeds the luminosity of any other type of AGN (excluding blazars, where it is posited that the intense luminosity is due to a powerful radio jet being aligned with the line-of-sight with which they are being viewed, which produces relativistic beaming effects). In some ways, the definition of the quasar category is due to outdated methods of categorization. Initially the high-z bright objects were 
identified in the radio as being brilliant sources without optical counterparts other than what looked like a particularly blue star. But the current paradigm allows for a range of radio brightnesses for quasars, or rather often allows the direct comparison of quasars with QSOs, which are not radio loud (it is this paradigm I adopt, and unless otherwise indicated this dissertation uses QSO and quasar interchangeably). The requirement that quasars are point-sources, or quasi-stellar - that is, that they do not show any signs of being part of a galaxy - is also obsolete as modern technology, in particular the Hubble Space Telescope, has revealed the host galaxies of select nearby quasars. In addition, in the past twenty years a class of dust-obscured quasars has been revealed, whose objects emit no extreme optical luminosity due to dust that shrouds the emission of the nuclear region. So the modern definition of quasars requires neither high optical luminosities nor high radio fluxes nor point-source objects. Instead, quasars are defined as being dominated by their AGN emission, which can be determined using SED modelling and color-color diagrams. In particular, color-color diagrams are commonly used in the IR to compare the near-, mid-, and far- infrared slopes to determine the significance of an object's AGN (Cutri et al. (2002), Zakamska et al. (2003), Lacy et al. (2004), Stern et al. (2005), Donley et al. (2005), Lacy et al. (2007a)).

\subsection{The Role of Environment (The Quasar-Galaxy Evolution Relationship)}

Studies of quasars have revealed a variety of interesting characteristics, not the least of which is that they seem to occur in merging systems. Not all AGN are located in obvious mergers, but the brightest and most massive are often found in morphologically 
disturbed host galaxies (e.g., Sanders \& Mirabel (1996), Bennert et al. (2008), Urrutia et al. (2008)). Studies of AGN, especially quasars, in morphologically interaction systems reveal an evolutionary sequence: when massive spiral galaxies interact, they pass through several stages of "disturbance" during which their gas is either heated and stripped, has its angular momentum transferred out into other parts of the system so it may fall to the gravitational center of the merger, or is shocked into forming stars. This fuels or activates the $\mathrm{SMBH}(\mathrm{s})$ of the merging system and drives large quantities of star formation (often in the form of massive starburst events). Star formation and AGN activity consume or blow out the gas until it is predominantly used up, leading to a gas-depleted, dispersion-dominated, structureless elliptical galaxy. In other words, through the merger process, massive spirals evolve to become elliptical galaxies. In this scenario, quasars and ultra luminous infrared galaxies (other brilliant, possibly-AGN-dominated class of sources) are short-lived stages in the life of evolving galaxies, and during other stages the SMBH is less luminous, perhaps even falling outside the classification of an AGN at certain points (Sanders et al. (1988c),Lonsdale et al. (1995), Hopkins et al. (2008), Treister et al. (2010)).

While this scenario is concise and erudite, it clearly is challenged by observations, and makes the connection between evolving galaxies and different types of AGN very much muddled. The brightest AGN do tend to be found in galaxies with morphological signs of current or past merging; that is, the brighter the AGN, the more likely it is to be found in such a system (e.g. Treister et al. (2012)), suggesting that mergers are a key to producing the most luminous or extreme AGN, but not a requisite for AGN activity. But not every bright AGN is found in a merger, and the selection method for determining "AGN" has an effect on whether or not the luminosity dependence is observed (Villforth et al. (2014)). This leads me to a phenomenon I call the "extrema 
effect" - the idea that the most luminous objects, which are the easiest to identify and study, might provide a false correlation for the overall AGN population.

The environment also contributes to the presence of dust, which has the potential to obscure the dominant power source within objects that might otherwise be considered as high luminosity. Since mergers produce a great deal of dust as well as star formation, according to the evolutionary scenario described above, quasars undergo a dust-obscured and unobscured phase within an evolutionary sequence. However, this is not the only way for quasars to be obscured by dust. According to models (Antonucci (1993), Urry \& Padovani (1995), Ueda et al. (2007)), on the outskirts of a SMBH's accretion disk is a toroid of thick dust that can block the intense luminosity of SMBH from reaching observers depending on the angle at which the object is viewed. The benefit of this scenario is that it is relevant no matter the luminosity of the AGN. For example, Seyfert galaxies, which contain AGN signatures but are not nearly as luminous as quasars or QSOs, fall into different categories depending on what sorts of emission lines are visible in their spectra, with Seyfert 1 having both doppler-broadened (1000s of $\mathrm{kms}^{-1}$ ) permitted emission lines and narrower (100s of $\mathrm{kms}^{-1}$ ) forbidden and permitted emission lines, and Seyfert 2 having solely the narrower lines. However, studies of polarized flux from the center of Seyfert 2 galaxies reveal broad Seyfert 1-like emission lines, obscured from the direct line of sight, but reflected (and thus polarized) by dust in the nuclear region slight further from the SMBH, suggesting a high opacity obscuration that does not cover the entire nuclear region - hence, a toroid. Quasars are more luminous than Seyfert galaxies, but a dusty nuclear toroid could still cause the difference between obscured and unobscured quasars - it simply needs a sufficiently high opacity. Observational work is often framed as pitting the orientation/torus model against the evolutionary scenario 
model, but there is no reason why both effects could not play a role in determining the characteristics of the quasar population and even within an individual AGN.

\subsection{The Complexity of AGN (Multiple Levels of Understanding Quasars)}

Overall, our understanding of AGN in general and quasars in particular is shaped by how we study them - how we account for differences in intrinsic AGN properties, in environmental effects on AGN properties, and in observational effects on our interpretations of AGN properties.

The first level is to study the distribution of the inherent characteristics of the SMBH itself. Most obviously this refers to differences in mass or density, spin, Schwarzschild radius, luminosity, and radiative efficiency. However it also encompasses such other features as the density and scale-size of the accretion disk, and the presence and characteristics of AGN jets. Although it is unclear which of these properties play the most significant role in determining an AGN's characteristics, it is most usual to consider the radiative efficiency (often sorted into low or high Eddington accretion rates), luminosity, or radio-loudness. Exploring these properties is the goal of the study of active SMBH, but such study is inherently entangled with the effects of observations and environment.

The second level is to explore the environment in which the quasar is found. This includes:

- the immediate nuclear environment of the SMBH, on scales from $\sim 1$ AU to $\sim$ 10s of pc:

- the opacity, size, opening angle or scale height, and uniformity (or lack 
thereof) of the dusty torus

- the size and density of the accretion disk

- the presence, whether in the accretion disk, dusty torus, or as clouds of various size scales and compositions, of atoms and molecules capable of being ionized, heated, or excited to cause broad or narrow emission lines

- the presence of gas clumps dense enough to form knots of young star formation, and the winds, outflows, and radiation that such star formation produces

- the presence, power, and extent of a nuclear jet that produces synchrotron radiation, or any other form of outflow, and the interaction between such an outflow and the nuclear material, which may produce shocks or other energetic signatures

- the circumnuclear environment, on scales of 0.1-3 kpc:

- the presence of circumnuclear star formation and its relation to driving infalling gas further in to the nuclear region for fueling the $\mathrm{SMBH}$, or alternatively blowing infalling gas out of the region, turning off further nuclear activity

- the presence of gas and molecular clouds to produce further emission and absorption of the nuclear emission, and to interact with nuclear outflows or jets

- the extent, distribution, opacity, and uniformity (or lack thereof) of circumnuclear dust and its ability to reprocess UV and optical light into the IR

- the host galaxy environment, on scales of 10s-100s kpc: 
- the extent and distribution of gas and stars within the host galaxy, characterized by galaxy type and the large scale SED of the galaxy, and

- the overall mass of the host galaxy, and how that mass is distributed in terms of dark matter, gas, and stars

- the extent and distribution of dust

- any interactions or mergers occuring in the nearby environment, both large scale (equal-mass) and small-scale (consuming a satellite or dwarf galaxy)

- the environment of the halo in which the quasar is located, including whether it is found to be part of a cluster, a group, or more ambiguously in the "field", and how that environment evolves with time

- the quasar's position in the cosmic web and the redshift or age at which it is being observed

All of these effects can modify, obscure, or otherwise complicate the inherent characteristics we are interested in from the first level of understanding.

The third level of understanding is to investigate the effects of observational parameters. As humans we are limited both in time and in space - we can observe for only our short human lifespan, a flicker of time in the life of a quasar. Neither can we emerge from our galaxy to observe SMBH from all angles, at all size scales. And we cannot create different quasars with varying mass and explore how they appear experimentally - we can only rely on observations to create statistically significant samples, or curious individual objects to suggest the effect of a property that we have not yet considered.

It is this concept of vast variation in properties, environment, and observational techniques that shapes the cutting edge of quasar research today, and leads to the 
fundamental questions that overarch this dissertation, as well as the more specific questions that I seek to answer.

\subsection{Questions Underlaying This Dissertation}

Within this dissertation, I present research that addresses the following specific questions:

- How does the host galaxy environment compare for obscured and unobscured quasars? And is there evolution with redshift? (Chapter 3)

- How does star formation contribute to the luminosity of the circumnuclear region of less-luminous SMBH? how does this affect fueling processes? And at what luminosity and size scale is the contribution from an AGN difficult to distinguish from bursts of star formation? (Chapter 2)

questions I explore in proposals for future work (Chapter 4):

- What is the morphological structure of quasar host galaxies? Is there evidence for mergers or interactions at a range of redshifts, for both obscured and unobscured quasars?

- What does the broad SED of quasar host galaxies tell us about the role of dust obscuration in the host-galaxy level environment?

- What are the environments like of AGN that demonstrate active feedback with their host galaxies? 


\section{Chapter 2}

\section{High Resolution Mid-Infrared Imaging of the Active Regions in Five Luminous Infrared Galaxies}

\subsection{Abstract}

High spatial resolution mid-infrared observations taken with the Very Large Telescope are used to explore the circumnuclear structure in a sample of five luminous infrared galaxies (LIRGs). Data taken in narrow-band VISIR filters at 12.81 and $13.01 \mu \mathrm{m}$ reveals the mid-infrared structure at a resolution of $\sim 100 \mathrm{pc}$ within the central 1-2 kpc of each LIRG. The five LIRGs show diverse circumnuclear morphology. Two are centrally dominated point sources, with little diffuse structure detectable above the background. Three of the objects have extended emission on scales of $0.1-2 \mathrm{kpc}$, and one of these contains a circumnuclear spiral For four of the five LIRGs, circumnuclear $(\sim 0.5 "$ scale $)$ mid-infrared flux is on order of $1 \mathrm{Jy}$, the same level at which the Infrared Astronomical Satellite (IRAS)-detected $12 \mu \mathrm{m}$ fluxes for the galaxy as a whole, on 
arcminute scale. The rarity and significance of a circumnuclear spiral in an early-type galaxy is discussed, as well as the role it could play in fueling the supermassive black hole.

\subsection{Introduction}

Luminous and ultraluminous infrared galaxies (LIRGs: defined as $L_{I R}[8-1000 \mu \mathrm{m}] \geq$ $10^{11.0} \mathrm{~L}_{\odot} ;$ ULIRGs: $\left.L_{\mathrm{IR}} \geq 10^{12.0} \mathrm{~L}_{\odot}\right)$ are primarily mergers of gas-rich galaxies. During the interaction, molecular gas is driven into the nuclear region where circumnuclear star formation, and sometimes active galactic nuclei (AGN) activity, is turned on. The resultant star formation rates can be as high as a few hundred solar masses per year, the bulk of which occurs within the central few kiloparsecs of the merging system. As a result, U/LIRGs are ideal systems for studying the properties of star formation in extreme environments (e.g., Sanders \& Mirabel (1996); Kennicutt \& Evans (2012)).

Assessing the nature of star formation in U/LIRGs is complicated by the high levels of dust obscuration towards their nuclear regions. While optical Hubble Space Telescope $(H S T)$ studies of U/LIRGs reveal star formation via the presence of UV/opticallyluminous star clusters (e.g. Surace et al. (1998); Farrah et al. (2001); Evans et al. (2008); Inami et al. (2010); Modica et al. (2012)), these studies cannot detect highly extinguished clusters in the nuclear regions where most of the star formation is occurring. The alternative is the mid-IR, as the opacity of dust drops with increased wavelength. This means that studies such as the broadband mid-IR imaging work by Alonso-Herrero et al. (2006); Alonso-Herrero (2013) and Díaz-Santos et al. (2007b), hold the promise of effectively probing nuclear star formation in all but the most extremely obscured nuclei. This work presents narrowband imaging at 12.81 and 13.01 
$\mu \mathrm{m}$ in the circumnuclear regions of five local $(z<0.03)$ LIRGs in order to assess the distribution of embedded star-forming regions. The data in this work were acquired using the European Southern Observatory's Very Large Telescope (VLT) and its VLT Imager and Spectrometer for mid-Infrared (VISIR) instrument. The VISIR on VLT makes it possible to achieve the high resolution required to resolve the morphological structure at scales of $100 \mathrm{pc}$ within the central 1-2 kpc.

The observations and the data reduction are summarized in Section 2.3. The observational parameters, including resolution in arcseconds and parsecs, are given in in Table 2. Section 2.4 presents the data, including the host galaxy classification and the nuclear morphology. Results are discussed in Section 2.5, including an exploration of the rarity of circumnuclear spirals and their implications, and the diversity of brightness surface density among the sample.

A cosmology with $\Omega_{\mathrm{M}}=0.3, \Omega_{\Lambda}=0.7$, and $H_{0}=70 \mathrm{~km} \mathrm{~s}^{-1} \mathrm{Mpc}^{-1}$ is used throughout.

\subsection{Sample, Observations, and Data Reduction}

\subsubsection{The Sample}

A sample representative of local LIRGs with available ancillary Spitzer data was chosen for this study based on redshift, IRAS $12 \mu \mathrm{m}$ flux and visibility from the site of the VLT in Chile. The selection was chosen with the initial goal of having the $[\mathrm{Ne}$ II] $12.8 \mu \mathrm{m}$ emission line redshifted into one of the VISIR narrow band filters. To focus on resolvable luminous objects, the IRAS $12 \mu \mathrm{m}$ flux of the objects was required to be greater than $250 \mathrm{mJy}$, with high-resolution broad and narrow-band HST images available. Nine LIRGs were selected for the original sample from a Spitzer survey (PID 
3672: PI J. M. Mazzarella) of a complete sample of LIRGs in the IRAS Revised Bright Galaxy Survey (RBGS: Sanders et al. (2003)); however, observations were limited by poor weather and only five of the nine selected objects were observed. A summary of the final sample is given in Table 2.1.

\subsubsection{Observations}

Mid-infrared band images were obtained from the European Southern Observatory Very Large Telescope (VLT) using the VLT Imager and Spectrometer for mid-Infrared (VISIR) instrument during 2006 January 17-18. The detector is a Si:As $256 \times 256$ array and the intermediate field (with a pixel scale of $0.127 /$ pixel) was chosen, yielding a field of view of $32.5^{\prime \prime} \times 32.5^{\prime \prime}$. A parallel chop-nod technique was used during observations, generating two negative and one positive beam on the detector. The chop-throw was set to 10", thus reducing the effective field of view to about a third of the original, i.e. to approximately 10 " x 10 ". The VISIR images presented in this paper therefore cover only the very center of each galaxy.

Two images were obtained of each galaxy nucleus (except MCG-03-34-064) in the 12.81 and $13.01 \mu \mathrm{m}$ filters. The filters have half-band widths of 0.21 and $0.22 \mu \mathrm{m}$, respectively. Total on-source integration times varied between $30 \mathrm{~min}$ and $1.5 \mathrm{hrs}$ and are listed in Table 2 together with relevant information about the observations. The diffraction limit of the VLT at $12.81 \mu \mathrm{m}$ is $\theta=1.2 \lambda / d \sim 0.39^{\prime \prime}$, and, as seen in Table 2, the galaxy images have resolutions close to the diffraction limit. Because of the longer integration times coupled with changing conditions during integration, the diffraction limit was not quite reached for the science targets. MCG-03-34-064 was only observed with the $13.01 \mu \mathrm{m}$ filter.

Conditions were clear on both nights, although with more sky variability and rel- 
atively high humidity $(\sim 30 \%)$ during the first night. Standard stars from the list by Cohen et al. (1999) were observed throughout in order to monitor the photometry and the shape of the point spread function (PSF), and perform flux calibrations. The variability in the flux (standard deviation per unit flux) of these standard stars over the course of the observations remained below $3 \%$ throughout the evening and proved more dependent on filter than on time, indicating a minimal effect on observations. The standard Poisson uncertainty on the counts is $<1 \%$ for all observations of standard stars, well below the level of variation described above.

\subsubsection{Data Reduction}

The raw data were processed through the ESO pipeline (version 'visir/1.3.7'), which builds the average of all chopped frames for each telescope nodding position. The different nodding frames are thereafter combined to produce a final image with two negative and one positive beam. Final images were made by adding the three beams by first multiplying the two negative images by -1 , aligning them with the positive image, and thereafter adding all three. As the positive beam contains half the total flux and the two negative ones each a quarter of the total flux, the final added image contains the total flux of the source within this aperture.

\subsection{Results}

\subsubsection{Overview}

Below, the VLT VISIR images are presented, and the morphology of each object is discussed. Three out of the five sources have a definitive central point source, presumably corresponding to an AGN or a compact nuclear starburst. The galaxies 
Table 2.1. The Sample

\begin{tabular}{cccccc}
\hline \hline Galaxy & IRAS identifier & z & $\begin{array}{c}\text { RA } \\
\text { hh:mm:ss.s }\end{array}$ & $\begin{array}{c}\text { Dec } \\
\text { dd:mm:ss.s }\end{array}$ & \multicolumn{1}{l}{$\log \left(L_{8}-1000 \mu \mathrm{m} / L_{\odot}\right)$} \\
\hline ESO 420-G013 & F04118-3207 & 0.011908 & $04: 13: 49.69$ & $-32: 00: 25.1$ & 11.07 \\
NGC 2369 & F07160-6215 & 0.010807 & $07: 16: 37.73$ & $-62: 20: 37.4$ & 11.16 \\
NGC 4418/4355 & F12243-0036 & 0.007268 & $12: 26: 54.62$ & $-00: 52: 39.2$ & 11.19 \\
MCG-03-34-064 & F13197-1627 & 0.016541 & $13: 22: 24.46$ & $-16: 43: 42.9$ & 11.28 \\
NGC 5135 & F13229-2934 & 0.013693 & $13: 25: 44.06$ & $-29: 50: 01.2$ & 11.30
\end{tabular}

Note. - $L_{F I R}$ from Armus et al. (2009)

Table 2. Summary of Observations

\begin{tabular}{ccccc}
\hline \hline Galaxy & $\begin{array}{c}\text { Int time (s) } \\
12.81 \mu \mathrm{m} \text { filter }\end{array}$ & $\begin{array}{c}\text { Int time (s) } \\
13.01 \mu \mathrm{m} \text { filter }\end{array}$ & Observing dates & $\begin{array}{c}\text { Resolution } \\
\text { arcsec (pc) }\end{array}$ \\
\hline ESO 420-G013 & 2600 & 3600 & $2006-01-18$ & $0.45(110)$ \\
NGC 2369 & 4048 & 5532 & $2006-01-17 \&-18$ & $0.50(111)$ \\
NGC 4418 & 1800 & 1800 & $2006-01-17$ & $0.49(73)$ \\
MCG-03-34-064 & $\ldots$ & 1840 & $2006-01-17$ & $0.42(141)$ \\
NGC 5135 & 3600 & 3600 & $2006-01-18$ & $0.44(123)$ \\
\hline
\end{tabular}

Note. - The second and third columns list on-source integration times for the two filters. Note that there is no $12.81 \mu \mathrm{m}$ image for MCG-03-34-064; only a $13.01 \mu \mathrm{m}$ image was obtained. The resolution in units of arcsecs (and parsecs) in each field is given in the last column. 
without a secure central point source detection are NGC 2369 and NGC 5135, in which there are knots embedded in more extended emission; it is not possible with these data to distinguish which of these, if any, might be considered a central source. Two of the galaxies do not show evidence for extended emission, whereas three show extended emission across the inner kiloparsec. A nuclear spiral has been discovered in one galaxy, ESO 420-G013. Flux values and uncertainties for each filter are summarized in Table 3.

\subsubsection{ESO 420-G013}

ESO 420-G013 is classified by the de Vaucouleurs et al. (1991) Third Reference Catalogue of Bright Galaxies (hereafter RC3) as a SA0+(r)pec type galaxy; by the Lauberts (1982) ESO/UPPSALA Survey of the ESO(B) Atlas (hereafter ESO/U Survey) as a S0(r) type galaxy; and by the Loveday (1996) APM Bright Galaxy Catalog as an E type galaxy. Though there is diversity in prior classifications, the consensus is that it is an early type lenticular or elliptical galaxy, likely with a ring. Maia et al. (1996) classify ESO 420-G013 as an optical Seyfert 2.

As shown in Fig 2.1, the circumnuclear region has an extended morphology in the MIR. Spiral structure is evident, with two prominent arms, each extending $\sim 700$ pc radially in the northeast and the southwest directions. A minor filament extends $\sim 500$ pc to the south. A central point-like source is evident.

\subsubsection{NGC 2369}

The galaxy NGC 2369 is classified by the RC3 as a SB(s)a type galaxy; by the ESO/U Survey as a Sa type galaxy; and by the Sandage \& Tammann (1995) Revised ShapleyAmes Catalog of Bright Galaxies (hereafter RSA) as a S(s)bc pec type galaxy. Again, 


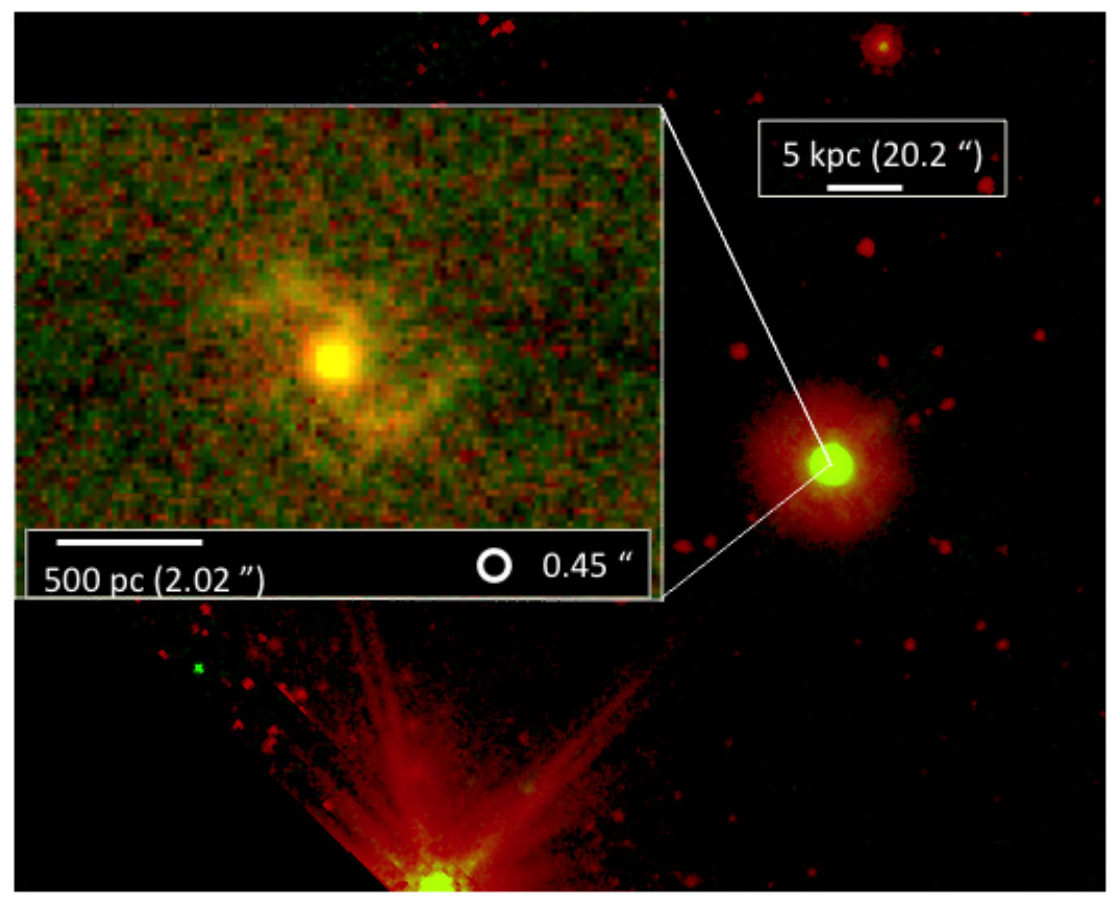

Fig. 2.1. - Images of ESO 420-G013. The host galaxy observed with Spitzer IRAC (red: channel 2, $4.5 \mu \mathrm{m}$; green: channel 4, $8.0 \mu \mathrm{m}$ ) is displayed with an inset of the circumnuclear region observed with VLT VISIR (red: $12.81 \mu \mathrm{m}$ filter; green: 13.01 $\mu \mathrm{m}$ filter). In both images, north is up, east is to the left, and corresponding scale bars are shown. 
there is variety in the classifications, but the consensus is that it is an early type spiral galaxy, probably with a bar. There is no published evidence that NGC 2369 has an active nucleus.

As shown in Fig 3.1, this circumnuclear region also contains extended morphology. Bright knots are slightly more prominent against the extended emission in the 12.81 $\mu \mathrm{m}$ filter; however both filters feature two obvious point-like sources, one to the north of the image (region A) and one to the south (region B), separated by $\sim 650$ pc. The more diffuse extended emission spans $\sim 1.4 \mathrm{kpc}$, slightly inclined to the west of true North-South, and encloses both regions. Two smaller knots are visible, $\sim 250$ and $200 \mathrm{pc}$ west of A. One possible interpretation of the morphology of this object is that it is a highly-inclined nuclear ring or spiral; if this is the case the minor axis would be $\sim 330 \mathrm{pc}$, and, assuming such a ring would be essentially circular, that yields an inclination $i \approx 76^{\circ}$ from edge-on or $i \approx 14^{\circ}$ from face-on. Although two point sources could indicate a double nucleus, the host galaxy has highly symmetric spiral arms, making it unlikely to be in such an advanced merger stage. Since there is evidence for a bar, the two knots may mark giant HII regions (often found at opposing ends of a stellar bar: see, e.g., Schinnerer et al. (2006), and references therein).

In a near-infrared imaging study by Piqueras López et al. (2012) and 2013 (hereafter PL12, PL13), similar complex morphology is identified in the $\operatorname{Br} \gamma$, [Fe II], and $H_{2}$ emission from NGC 2369 (see PL12, Fig. 1a). The $\operatorname{Br} \gamma$ and $H_{2}$ velocity contours presented therein indicate rotation, and the authors suggest this indicates a warped rotating disk, confirming the interpretation of an inclined ring, spiral, or generally disk-like circumnuclear structure within NGC 2369. While the $\mathrm{Br} \gamma$ and [Fe II] emission maps in PL12 generally traces the same, albeit more diffuse, structure as the observations presented here, the $H_{2}$, K-band continuum, and HST F160W images in 


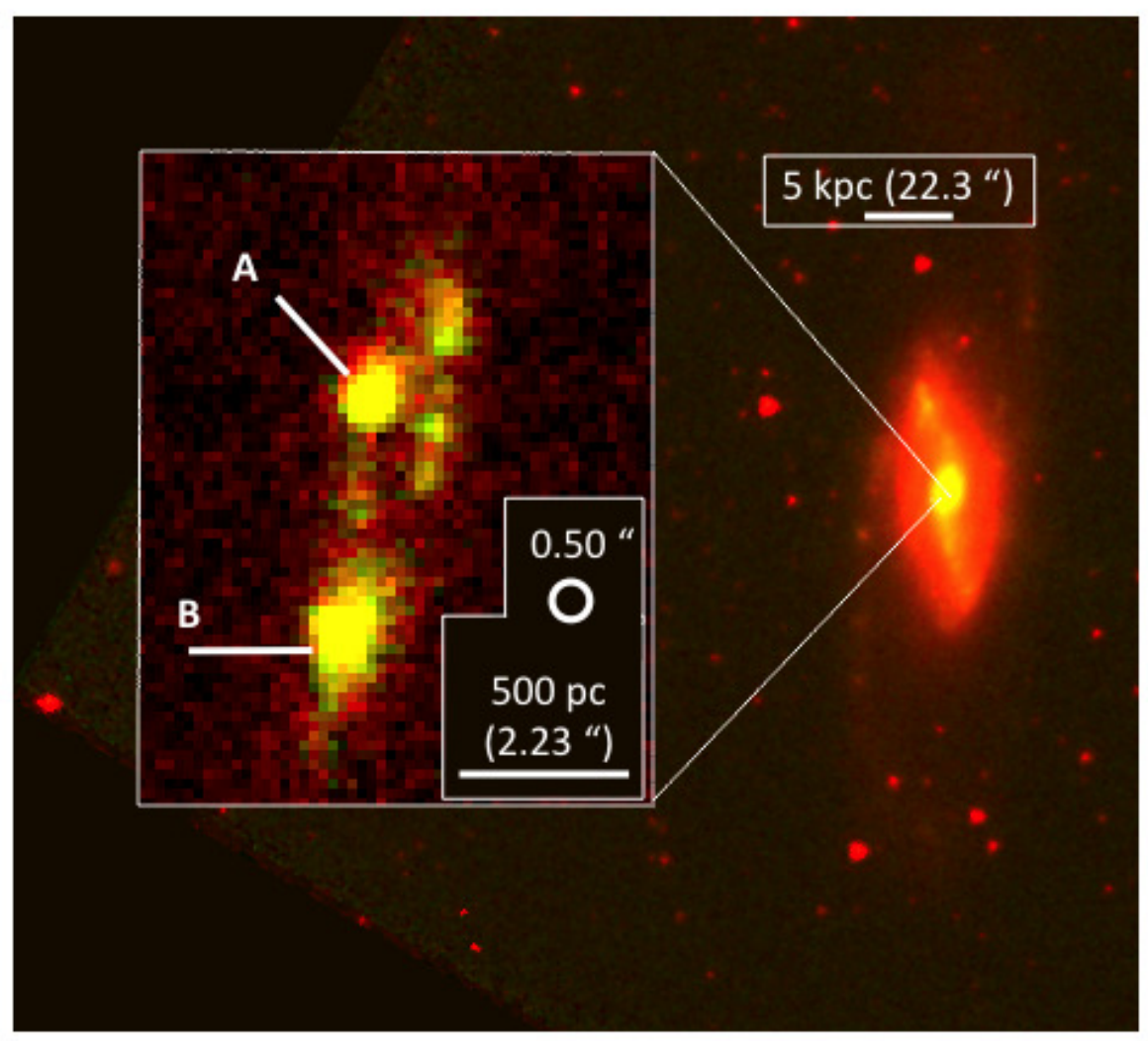

Fig. 2.2. - Spitzer IRAC and VLT VISIR images of NGC 2369, as described in the caption of Figure 1. 
PL12 show a different morphology. Region A in Fig 3.1, especially, is not identified in $H_{2}$ or either continuum map, confirming this as a detection of a deeply embedded region. In PL13, where a map of $A_{v}$ (extinction) is plotted for NGC 2369 (see PL13, Fig. 1a), the region corresponding to Region A in Fig 3.1 does not show particularly strong extinction compared to the rest of what might be considered part of a circumunuclear disk, suggesting an equivalent opacity throughout such a disk.

\subsubsection{NGC 4418}

NGC 4418 is classified by the RC3 as a (R')SAB(s)a type galaxy, by the Nilson (1973) Uppsala General Catalogue of Galaxies as a Sa type galaxy, and Naim et al. (1995) as either a $\mathrm{Sa}, \mathrm{S}^{+}$or $\mathrm{S} 0 /$ a type galaxy. Véron-Cetty \& Véron (2006) categorize its nuclear activity as Seyfert 2 based on the lack of broad lines and the $[\mathrm{O} \mathrm{III}] / \mathrm{H} \beta$ ratio.

As shown in Fig 2.3, this object has no extra-nuclear MIR emission visible with the given resolution limit of $\sim 75$ pc. In a radio imaging study by Costagliola et al. (2013), they estimate a hot, compact core on order of 5 pc, suggesting a deeply embedded AGN or starburst equivalent to that found in Arp 220. They also identify in-flow in both the HI absorption line and in the CO 2-1 emission, attributing its source to the interaction between NGC 4418 and its companion. No signature of such an inflow is visible in the MIR data presented here.

The compact nucleus, combined with the deep silicon absorption evident in archival spectra information (see Section 2.5.1) support the idea that this object hosts a deeply embedded AGN. 


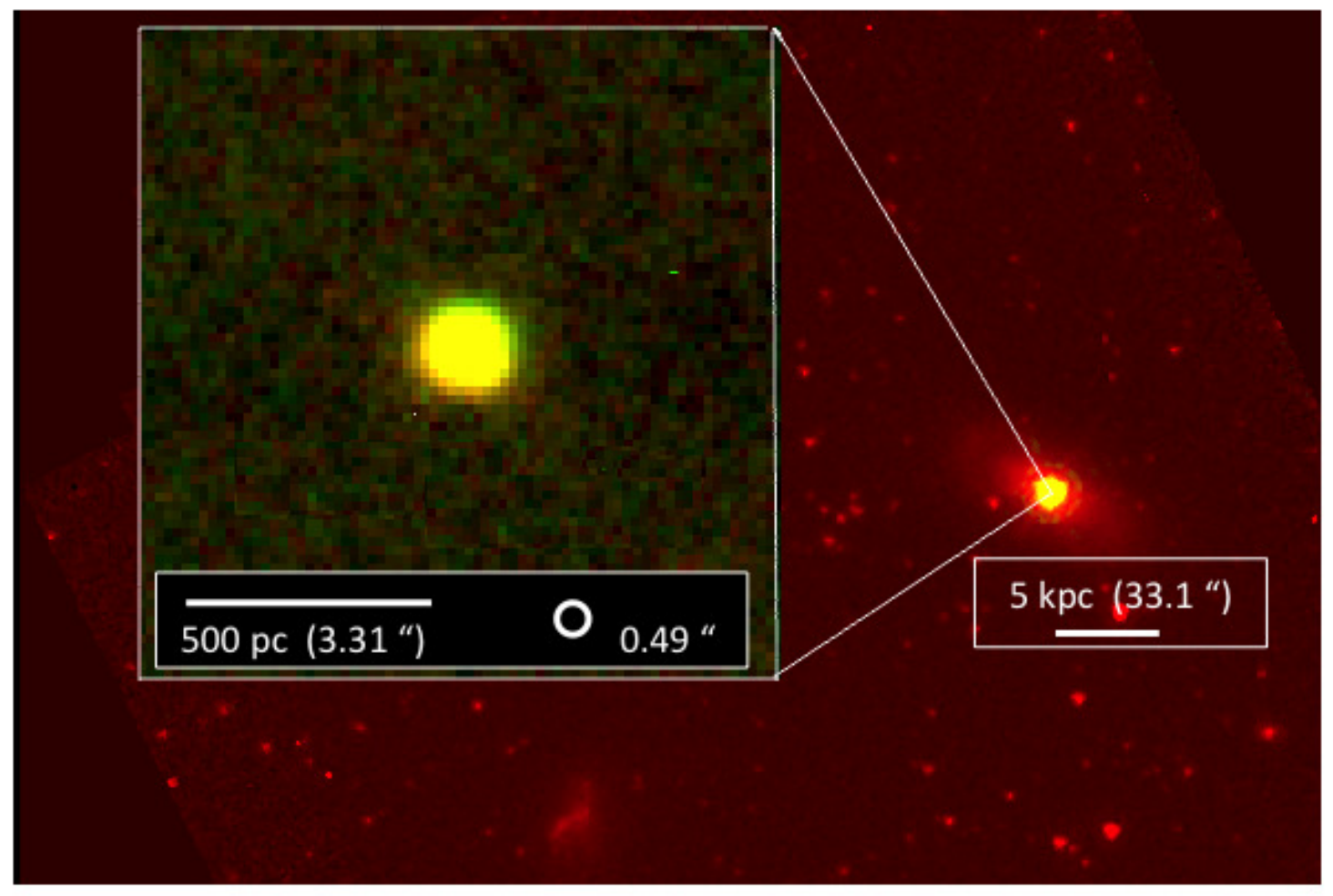

Fig. 2.3.- Spitzer IRAC and VLT VISIR images of NGC 4418, as described in the caption of Figure 1. 


\subsubsection{MCG-03-34-064}

MCG 03-34-064 is classified by Naim et al. (1995) as either a $\mathrm{S}^{+}$or a $\mathrm{S} 0 / \mathrm{a}$, and thus it appears to be a late-type lenticular galaxy. Véron-Cetty \& Véron (2006) categorize its nuclear activity as a Seyfert 1h; i.e., a Seyfert 2 with Seyfert 1 spectral characteristics when viewed with polarized light.

The nuclear region of MCG 03-34-064 shows no extended morphology, only a simple central source (see Figure 2.4). Note that the PSF of the standard stars also occasionally demonstrated a slight elongation throughout the nights of observation.

\subsubsection{NGC 5135}

NGC 5135 is classified by the RC3 as a SB(s)ab type galaxy, by the RSA as a $\mathrm{SBb}$ type galaxy, and by the ESO/U Survey as a Sa type galaxy. The consensus is thus that it is an early type barred spiral galaxy. Véron-Cetty \& Véron (2006) identify it by its nuclear activity as a Seyfert 2 galaxy.

As shown in Fig 2.5, this circumnuclear region also has an extended morphology, as well as a strong point source. This particular circumnuclear region has an irregular morphology, though like NGC 2369 it is feasibly a nuclear ring. The extended emission extends $\sim 1.4 \mathrm{kpc}$ from the northeast to the southwest and forms an arc. If this is assumed to be a ring, then the minor axis would be $\sim 750 \mathrm{pc}$, from the bright spot in the northwest to the more diffuse region in the southeast. The strong point source in the region labeled $\mathrm{A}$ in Fig. 2.5 is more prominent than the surrounding emission, and may be where the nucleus is located.

As NGC 5135 is one of the brightest LIRGs in the local Universe, it has been extensively studied even at high resolutions. Díaz-Santos et al. (2007a) spectroscopically differentiate between nuclear emission and HII knots in the central $\sim 2 \mathrm{kpc}$ of 


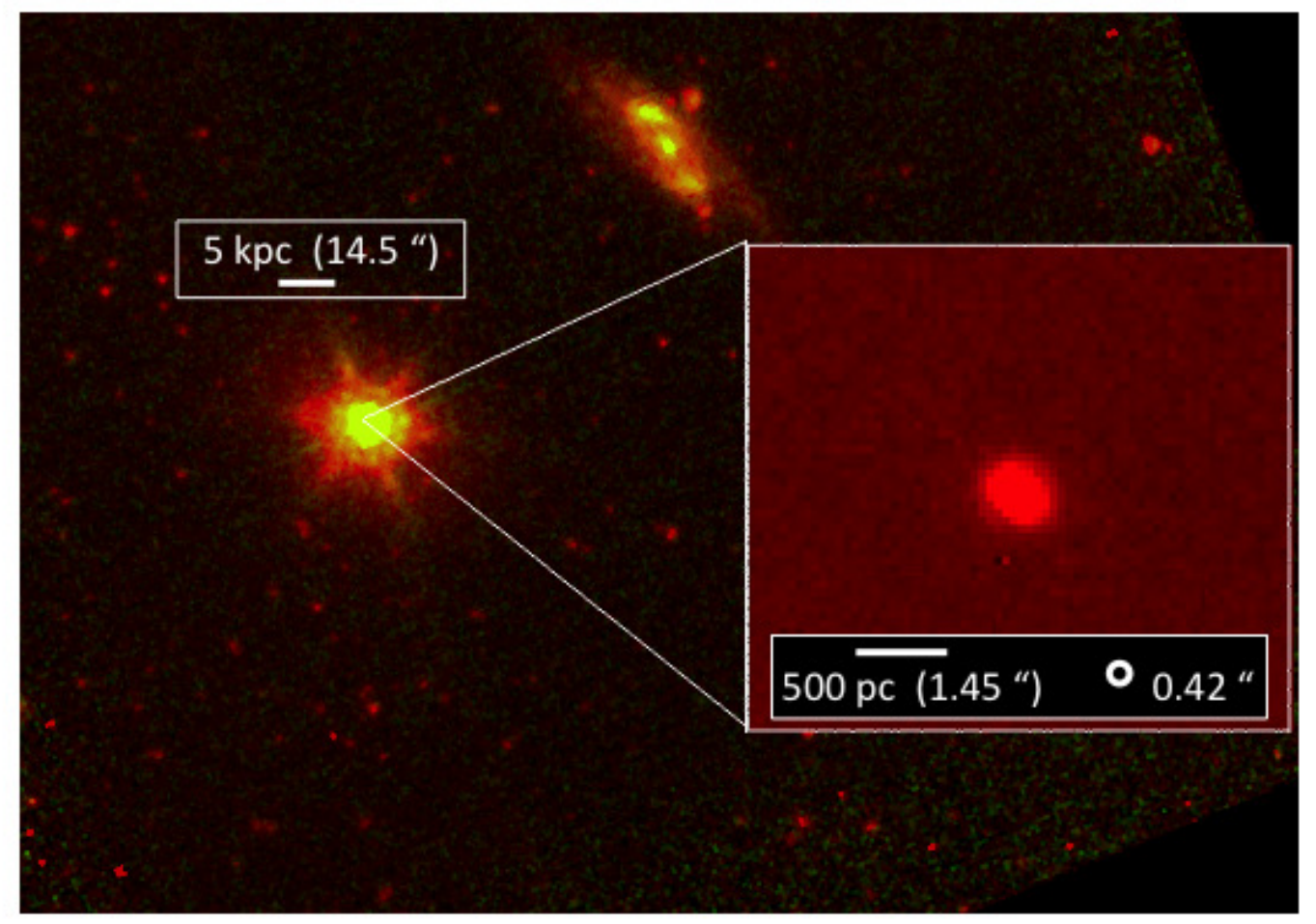

Fig. 2.4.- Spitzer IRAC and VLT VISIR images of MCG-03-34-064, as described in the caption of Figure 1. 


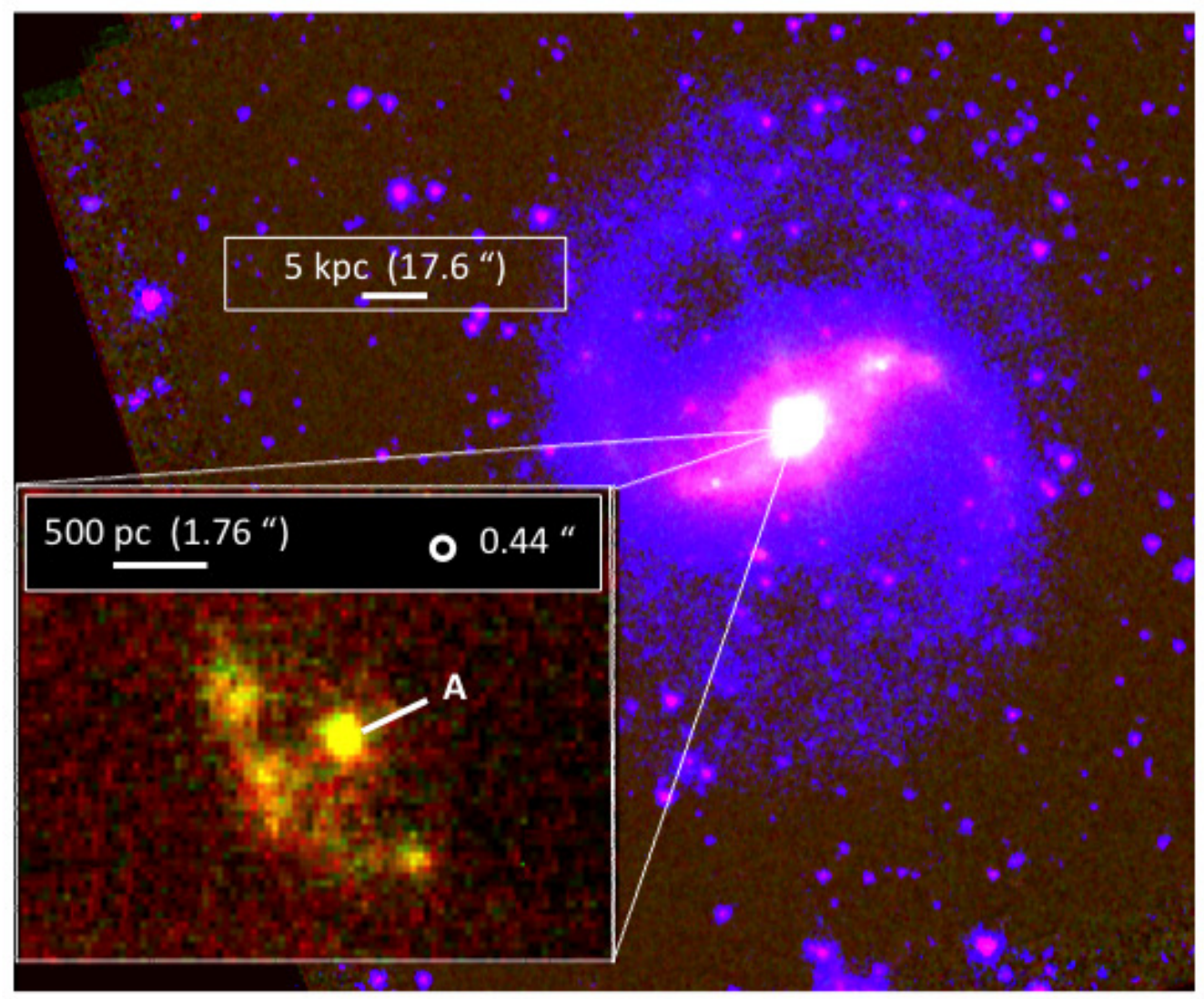

Fig. 2.5. - Spitzer IRAC and VLT VISIR images of NGC 5135, with blue: IRAC channel 1, 3.6 $\mu \mathrm{m}$ and the rest as described in the caption of Figure 1. 
NGC 5135. Horst et al. (2009) took data similar to that presented in this paper, using the $12.81 \mu \mathrm{m}$ filter of VISIR on the VLT - they also identify extranuclear emission that they attribute to intense star formation. Both PL12 and PL13 create circumnuclear maps of NGC 5135 using NIR integral field spectroscopy. They identify the region A in Fig. 2.5 (see Fig. 1f in either paper) in the K-band continuum and trace a similar, though more diffuse, structure of circumnuclear emission in $\mathrm{Br} \gamma$. However in their [Fe II] and $H_{2}$ emission maps, the primary feature is not region A but a bright spot to the southwest that does not appear in the $12.81 \mu \mathrm{m}$ image. This is likely a region of gas being warmed (and shocked, hence the [Fe II]) by either recent star formation or an outflow from the nucleus. Bedregal et al. (2009) and 2011 use the [Si VI] $1.96 \mu \mathrm{m}$ line to identify ionizing cones of coronal gas extending from the nuclear region (corresponding to region A of Fig 2.5) up to $600 \mathrm{pc}$ in projection. These contours appear spatially associated with the knot of $12.81 \mu \mathrm{m}$ filter emission to the northeast in Fig. 2.5, although they do not overlap.

\subsection{Discussion}

\subsubsection{Diversity of Nuclear Morphology}

The sample presented in this paper shows a range in morphological characteristics. Three objects have extended circumnuclear emission on the order of $\sim 1-2 \mathrm{kpc}$, but this varies between coherent structure (the spiral in ESO 420-G013 and possibly the ringlike structure in NGC 2369) and more chaotic structure (i.e., NGC 5135). Using high-resolution kinematic gas measurements may prove the solution to verifying the coherence of such structures, but such an investigation is beyond the scope of this paper. 
The two remaining members of the sample appear to be point-like at the $\sim 100$ pc resolution of the VLT observations; the compact structures are either AGN or nuclear star clusters (or a combination of the two). Spectral classifications indicate the presence of an AGN in both of them. Véron-Cetty \& Véron (2006), in the 12th edition of their catalogue of quasars and AGN, identify NGC 4418 as a Seyfert 2 galaxy and MCG 03-34-064 as a Seyfert 1h. This identification requires Balmer and forbidden lines of the same width for Sy2; to further classify the object as a Sy1h, Véron-Cetty \& Véron require the detection of a Sy1 spectrum in polarized light. However the presence of an AGN alone cannot account for their morphological simplicity, as ESO 420-G013 and NGC 5135 are also classified as Sy2.

Thus these two objects therefore can be said to have structureless circumnuclear environments at this wavelength, insofar as "circumnuclear" is defined as the region from $\sim 1 \mathrm{kpc}$ down to $\sim 100 \mathrm{pc}$, and can be said to have AGN present within that circumnuclear region. However this speaks more to the limitations of the observation than the characteristics of the circumnuclear regions of LIRGs, as there is likely unresolved structure at the sub-100pc scale. The galaxy NGC 4418 was the closest object in the sample, with $z=0.007268$ and 73 pc resolution; in contrast, MCG 03-34-064 was the farthest, with $z=0.016541$ and $141 \mathrm{pc}$ resolution. This spread in resolution limits unresolved structure to the $\sim$ tens of parsecs scale.

Finally, although it is unlikely, it is possible for extremely high dust column densities to obscure the star formation structures that were spotted in the other galaxies in these two "structureless" objects, even in the MIR. Spoon et al. (2001) investigated the MIR spectrum of NGC 4418 and found a featureless MIR continuum as well as deep absorption features, indicating high column densities, and they point out that "it would be highly unlikely to block the escape of any mid-IR starburst indicator 
from a region of that size", that is, the $\sim 70$ pc scale nuclear point source - thus they posit the presence of an enshrouded AGN.

The spectral information available for both sources with compact nuclei is particularly informative. The relatively flat slope of MCG 03-34-064, as well as the weakness of its MIR PAH lines (see, e.g. Stierwalt et al. (2013), Stierwalt et al. (2014), as well as Sargsyan et al. (2011)) suggest that this object is dominated by an AGN. The steep mid-infrared spectral slope of NGC 4418 (and the deep silicate absorption lines it displays in the circumnuclear MIR spectrum of Stierwalt et al. (2013)) are also indicative of an AGN, albeit a highly obscured one.

\subsubsection{Brightness Surface Density}

The flux density recovered in the majority of the high-resolution mid-infrared images from the VLT is on the order of 0.5-1 Jy. Strikingly, archival IRAS data at $12 \mu \mathrm{m}$ is on the same order of 0.5 - 1 Jy. Since the observations presented in this text cover the central $1 \mathrm{kpc}$ of each galaxy, this makes it probable that sufficient flux comes from the circumnuclear region to contribute significantly to the overall $\left.L_{(8-1000} \mu \mathrm{m}\right)$. Using a ratio of $12.8 \mu \mathrm{m}$ VISIR filter flux to $12 \mu \mathrm{m}$ IRAS flux, we derive a factor called $f_{12} \mu \mathrm{m}$, which describes the relative significance of the circumnuclear contribution to the total flux detected within the whole galaxy. For ESO 420-G013, NGC 2369, NGC 4418, and MCG 03-34-064, this ratio demonstrates relatively significant circumnuclear contributions. If we extrapolate that this implies the bulk of other MIR flux, and thus of the observed overall $L_{(8-1000 \mu \mathrm{m})}$, is also produced in these regions, we can put a lower limit on the Brightness Surface Density of the circumuclear regions. These data are included in Table 4, and range from $1.6-7.5 \times 10^{10} \mathrm{~L}_{\odot} k p c^{-2}$. For the two amorphous sources (ESO 420-G013 and NGC 2369), this calculation serves as lower 
Table 3. Photometry

\begin{tabular}{lllccc}
\hline \hline Galaxy & \multicolumn{2}{c}{$\begin{array}{c}\text { VLT flux } \\
13.01 \mu \mathrm{m} \text { filter }(\mathrm{Jy})\end{array}$} & $\begin{array}{c}\text { Area } \\
\text { arcsec }^{2}\end{array}$ & $\begin{array}{c}\log \left(\frac{L_{\text {band }}}{L_{\odot}}\right) \\
12.01 \mu 1\end{array}$ & $\mu \mathrm{m}$ Filter filter \\
\hline ESO 420-G013 & $0.52 \pm 0.015$ & $0.73 \pm 0.022$ & 125 & 8.23 & 8.38 \\
NGC 2369 & $0.84 \pm 0.025$ & $0.96 \pm 0.028$ & 62.1 & 8.35 & 8.41 \\
NGC 4418 & $1.6 \pm 0.048$ & $1.95 \pm 0.059$ & 93.0 & 8.28 & 8.37 \\
MCG 03-34-064 & $\ldots$ & $1.3 \pm 0.041$ & 90.5 & $\ldots$ & 8.92 \\
NGC 5135 & $0.031 \pm 0.00095$ & $0.049 \pm 0.0015$ & 102 & 7.13 & 7.33
\end{tabular}

Note. - No data in the $12.81 \mu \mathrm{m}$ filter were taken for MCG-03-34-064. Columns 2 and 3 are total summed fluxes within the filter bands from the VLT. Column 4 lists the area within which the flux was summed. Background subtraction was taken individually in each filter before the total fluxes were recorded. Uncertainties stem from a combination of Poisson noise, instrument and sky error, and calibration star flux variation throughout the night ( $\sim 3 \%$; see Section 2.2$)$.

Table 4. IRAS Fluxes and Luminosities

\begin{tabular}{|c|c|c|c|c|c|c|}
\hline Galaxy & $12 \mathrm{~J}_{\mathrm{y}} \mathrm{m}$ & $25_{\mathrm{Jy}}^{\mu \mathrm{m}}$ & $60 \mathrm{~J} \mathrm{~J}_{\mathrm{y}}$ & $100 \underset{\mathrm{Jy}}{\mu \mathrm{m}}$ & $f_{12 \mu \mathrm{m}}$ & $\begin{array}{c}\text { Brightness surface density } \\
\left(L_{\odot} k p c^{-2}\right)\left(10^{19}\right)\end{array}$ \\
\hline ESO 420-G013 & 0.95 & 2.22 & 13.66 & 20.88 & 0.55 & 1.6 \\
\hline NGC 2369 & 0.74 & 2.20 & 20.35 & 38.31 & 1.1 & 4.7 \\
\hline NGC 4418 & 0.99 & 9.67 & 43.89 & 31.94 & 1.6 & 7.5 \\
\hline MCG 03-34-064 & 0.94 & 2.97 & 6.2 & 6.2 & 1.4 & 1.9 \\
\hline NGC 5135 & 0.63 & 2.38 & 16.86 & 30.97 & 0.05 & $\cdots$ \\
\hline
\end{tabular}

Note. - Cols 2-5 are IRAS data from Sanders et al. (2003) of the flux values typically used to derive full $L_{8-1000 \mu \mathrm{m}}$, as in Armus et al. (2009). Col 6 is the "relative $12 \mu \mathrm{m}$ flux ratio" for determining circumnuclear significance (see Sec 2.5.2). 
limit estimate because the result is averaged over the area of both knots and diffuse emission that define the circumnuclear region. For the two point-like sources (NGC 4418 and MCG-03-34-064), the area is defined by a radius closer to the full-light radius than an effective or half-light radius. To increase accuracy of surface density for the extended objects requires exploring how much of the total flux in the $12.8 \mu \mathrm{m}$ image comes from knots as compared to diffuse structures.

Regardless, even as a lower limit, $\sim$ few $10^{10} L_{\odot} k p c^{-2}$ indicates significant density of star formation. Starburst galaxies such as M82 have characteristic surface densities of $\sim$ few $10^{11} L_{\odot} k p c^{-2}$ on average, with peaks of up to $10^{13} L_{\odot} k p c^{-2}$ in small $10 \mathrm{pc}$ scale knots or clusters such as those discussed in Section 1 (i.e., Soifer et al. (2001). In contrast, ULIRGs have significantly higher brightness surface densities on average, approaching and exceeding $10^{13} L_{\odot} k p c^{-2}$ in regions on the order of 100 pc. Such brightness surface density measures, and the scale of the knots or clusters in which they are measured, may serve as an indicator of the dominance of AGN or starburst processes in high luminosity objects, since the amount of luminosity per unit area able to be produced by star formation can be limited by our current observations of extreme starbursts (brightness surface densities higher than $10^{13} L_{\odot} k p c^{-2}$. The surface density measurements found in the 100-parsec scale regions for these four objects do not reach that extreme; this is consistent with star formation powering their high luminosity.

The exception in this case, NGC 5135, has a $12.81 \mu \mathrm{m}$ band flux of $0.031 \mathrm{Jy}$ and a $13.01 \mu \mathrm{m}$ band flux of $0.049 \mathrm{Jy}$, an order of magnitude lower than the IRAS $12 \mu \mathrm{m}$ flux of $0.63 \mathrm{Jy}$. There are several possible explanations for this discrepancy compared to the other LIRGs in the sample, but most significant is the morphology of NGC 5135. This barred spiral is nearly face-on, and the fluxes reported in the Revised 
Bright Galaxy Survey from IRAS are derived from a slit that encompassed on order of 1 arcmin, aligned along the bar. The bar by definition contains excess stars and can by itself contribute a significant portion of the total galaxy luminosity. In addition, the ends of bars are known to be sites of high star formation rates. So not only is the bar a strong MIR emitter, but with the large beam size of $I R A S$ there is a chance that additional regions of recent high mass star formation are incorporated into the data reported in Table 4.

\subsubsection{The Nature of the Circumnuclear Spiral found in ESO 420-G013}

Circumnuclear dust and gas spirals on the order of a few kpc have been predicted (e.g., Jogee et al. (2003); Ann \& Thakur (2005); Thakur et al. (2009); Maciejewski (2004a) and 2004b, Englmaier \& Shlosman (2000)), and detected (e.g. Walterbos \& Graeve (1985); Turner \& Hurt (1992); Colina et al. (1997); Laine et al. (1999); Chapman et al. (2000); Martini et al. (2003); Storchi-Bergmann et al. (2007); Davies et al. (2009)) in spiral galaxies for some time, often associated with and attributed to the presence of bars. In the case of ESO 420-G013, the host galaxy is an elliptical galaxy, not a spiral. Nuclear spirals in ellipticals are considerably rarer than those in spiral galaxies. According to the evolutionary scenario as outlined by Sanders et al. (1988c) or more recently by Hopkins et al. (2008), ellipticals are produced as the end stage of a major merger between two massive spiral galaxies. They do not typically contain stellar disk features and the SFR is generally low; most stars are older; hence the term "red and dead". However, mergers provide a means of transferring angular momentum from the gas, thus funneling it into the circumnuclear region of the resulting galaxy, 
where it can trigger star formation and fuel the AGN. Thus, it is reasonable to expect circumnuclear structure to be characteristically different from the larger scale (10s$100 \mathrm{~s}$ of $\mathrm{kpc}$ ) structure of the elliptical during the latest stages of the merger - the host galaxy has no gas left to fuel activity, but the circumnuclear region has sufficient gas to feed star formation and AGN activity.

We consider it edifying to compare and contrast ESO 420-G013 with two other elliptical galaxies in which circumnuclear spirals have been identified: Centaurus A and Arp 102B. Espada et al. (2012) uncovered a nuclear spiral in the giant elliptical galaxy Centaurus A (Cen A), which has both extensive radio jets and a closely-studied AGN (see, e.g., Beckmann et al. (2011)). There also exists evidence that Cen A is the product of a recent merger - most prominently, large scale $(r>6 \mathrm{kpc}) \mathrm{HI}$ gas streams that have not yet settled. Fathi et al. (2011) uncovered a nuclear spiral in the elliptical galaxy Arp 102B, which is a LINER/Seyfert 1 with a companion galaxy, Arp 102A. Both Fathi et al. (2011) and Couto et al. (2013) propose that jet-cloud interactions are responsible for triggering this nuclear spiral.

In comparison, ESO 420-G013 is an elliptical galaxy classified as a Seyfert 2. Its circumnuclear region contains a $\sim 1.4 \mathrm{kpc}$ spiral, a scale similar to the circumnuclear spirals of Arp 102B and Cen A. However, it is here where the similarities end. In currently available data, ESO 420-G013 has no signs of having recently undergone a merger. It has no equal-mass companion and does not show tidal streams (although the presence of smaller-massed companions is not yet determined due to lack of truly deep observations). Its luminosity peaks in the IR, suggesting it has a much higher dust content than either of the other two. This suggests that the circumnuclear spirals in these three objects either underwent different formation mechanisms or are at different stages in their evolution. 
The presence of a circumnuclear star-forming spiral in this object places certain restrictions on the characteristics of ESO 420-G013's nucleus. It implies that for this galaxy:

- gas is present, most likely in a disk structure with a scale size similar to the circumnuclear spiral.

- there is a spiral density wave present that can induce this star formation. Since the mid-infrared serves as a tracer of younger star formation and the scale size of the circumnuclear region is on order of a few kiloparsecs, a short-lived transitory spiral wave cannot be ruled out.

- a spiral density wave provides an opportunity for collisional shocks; thus, angular momentum is able to be removed from the gas, providing a means of funneling material down into the central tens of parsecs, potentially to feed the black hole.

As this spiral is embedded in an elliptical galaxy without obvious visible perturbations, it may even be more likely that a transient interaction would drive circumnuclear spiral structure, rather than a larger scale axisymmetric perturbation. With the dearth of evidence for a gas-rich major merger in any recent history of ESO 420-G013 (especially in comparison to other circumnuclear spirals in the literature), it is possible that its spiral structure would be driven by something other than a major merger. Keeping in mind that there are no truly deep images of this object, with sufficient sensitivity to spot low surface brightness emission, we suggest that ESO 420-G013 may have accreted a gas-rich satellite galaxy. In this scenario, the gaseous remnants of the satellite galaxy lost significant angular momentum and settled into the nuclear region, producing the spiral disk and fueling recent star formation in the process. 


\subsection{Conclusions}

The high resolution of the Very Large Telescope has been used to assess the dustshrouded circumnuclear regions of five local LIRGs and examine their small-scale $(<1 \mathrm{kpc})$ morphology down to $\sim 100$ pc. The following conclusions are proposed:

- Through high resolution imaging, we determine and report the 12.81 and 13.01 $\mu \mathrm{m}$ fluxes of $\sim 100$ parsec scale structures within the circumnuclear region. For four of these LIRGs, this flux density is on the order of $0.5-1 \mathrm{Jy}$.

- For the four high flux density objects, the $12.8 \mu \mathrm{m}$ filter flux is comparable to the total flux detected at $12 \mu \mathrm{ms}$ in the galaxy as a whole. indicating that the circumnuclear region often contributes a significant proportion of their IR emission. Extrapolating from this information, we calculate the infrared brightness surface density to range from $1.6-7.5 \times 10^{10} L_{\odot} k p c^{-2}$. These numbers serve as a lower limit estimate based on the extended distribution of flux in the circumnuclear region.

- The mid-infrared emission is not restricted to a central point source, but can take the form of more extended features. Morphologically, those extended features are diverse, and may contain kinematic information that relates to the process by which gas is driven all the way down to the accretion disk. The internal structures within the circumnuclear region, and the processes by how they form, may mirror those identified in host galaxies.

- A circumnuclear spiral has been identified within the elliptical galaxy ESO 420G013; it is $\sim 1.4 \mathrm{kpc}$ in diameter, and it is possible for it to have been triggered by the accretion of a gas-rich satellite galaxy. 
- In circumnuclear spectral data from other publications, NGC 4418 demonstrates deep silicate absorption and a relatively featureless spectrum. Combined with its compact circumnuclear nature, this suggests a deeply embedded AGN as the predominant source of its 12.81 and $13.01 \mu \mathrm{m}$ fluxes presented in this paper.

- The 12.81 and $13.01 \mu \mathrm{m}$ fluxes detected within NGC 5135's central kpc are both an order of magnitude lower than the total $12 \mu \mathrm{m}$ flux detected for the whole galaxy. We attribute this to the the presence of the bright bar in the host galaxy, where much additional high mass star formation might be triggered. The complex circumnuclear morphology of NGC 5135 suggests that recent star formation still plays a role in this region, as does the presence of shocks, while the detection of coronal gas cones simultaneously suggests an AGN component.

\subsection{ACKNOWLEDGEMENTS}

MW and KMJ thank the Dark Cosmology Center at the University of Copenhagen for hospitality and support. MW acknowledges the Research Council of Norway for support through grant number 177304. KMJ acknowledges the National Science Foundation for support through grant No. DGE-0809128, as well as the original VLT observing proposers H. Kaufl and R. Siebenmorgen. KMJ thanks S. Stierwalt for providing the CAFE-derived fitted parameters to the IRS spectra. ML acknowledges the National Radio Astronomy Observatory, which is a facility of the National Science Foundation operated under cooperative agreement by Associated Universities, Inc. This research has made use of the NASA/IPAC Extragalactic Database (NED) which is operated by the Jet Propulsion Laboratory, California Institute of Technology, under contract with the National Aeronautics and Space Administration. This work 
is based in part on observations made with the Spitzer Space Telescope, which is operated by the Jet Propulsion Laboratory, California Institute of Technology under a contract with NASA. 


\section{Chapter 3}

\section{Clustering in SERVS at}

\section{High-Redshift: No Difference in}

\section{Halo-Scale Environments between}

\section{Obscured and Unobscured Quasars}

\subsection{Abstract}

This work analyzes matched samples of obscured and unobscured quasars to characterize the megaparsec-scale environment of a previously unexplored sample of high redshift $(1.3<z<2.5)$ SIRTF Wide-field Infra-Red Extragalactic Survey (SWIRE) quasars. Optically obscured quasars are compared to a control sample of opticallybright quasars identified via selection in the mid-infrared using Spitzer colors as described in Lacy et al. (2007a). The SERVS data fields were observed at 3.6 and 4.5 $\mu \mathrm{m}$ to a depth of $\sim 2 \mu \mathrm{Jy}(\mathrm{AB}=23.1)$. The final high-z sample of 42 quasars $(18$ Type 1, 17 Type 2, and 7 Type 1R) is cross-correlated with the $\sim 4$ million galaxies 
from the Spitzer Extragalactic Representative Volume Survey (SERVS) filtered using color-color selection to remove low-redshift $(z<1.3)$ or foreground objects. Recent work has found diverse results in such studies, with variation of environmental richness by redshift, level of obscuration, sample selection, and luminosity of source. For this sample of quasars we find mean clustering lengths $\left\langle r_{o}\right\rangle=26.0 \pm 8.36$ and $18.0 \pm 7.12 \mathrm{Mpc}$ for Type 1 and Type 2 quasars, respectively. For the smaller sample of Type $1 \mathrm{R}$ we calculate $r_{o}=21.2 \pm 12.4 \mathrm{Mpc}$. Overall we find no statisticically significant distinction between the level of clustering for these different types of objects. Clustering amplitude also remains relatively independent of redshift within the range of the sample, indicating a consistent halo mass for quasar presence, which coincides with the findings of much recent work. I compare our results with diverse recent studies.

\subsection{Introduction}

Quasars are the most luminous SMBH-powered structures known, and provide a unique means for probing the specific role of an active nucleus in the formation and evolution of galaxies throughout cosmic history-a connection tantalizingly revealed by the black hole-bulge mass relation and the mass-and-dispersion relations uncovered at the turn of the century (e.g. Magorrian et al. (1998), Ferrarese \& Merritt (2000), Gebhardt et al. (2000)), and further explored by innumerable papers in the past 25 years. But there exists a large population of quasars that remains relatively unexplored - that of the dust-obscured quasars, also known as Type 2 quasars. These are traditionally difficult to detect, since they do not have the characteristic blue continuum and the Doppler-broadened emission lines that are some of the most prominent quasar signatures. It is not yet clear what it is that exactly leads to the 
differentiation between Type 1 (normal or unobscured) quasars and Type 2 (dusty or obscured) quasars, but what is clear is that a variety of factors can contribue to the distinction: 1) the orientation of we the observers with respect to the AGN's axis and/or accretion disk and/or jets, winds and outflows; 2) the presence of dust in the form of a nuclear torus, nuclear and circumnuclear diffuse or widely-distributed dust, and similarly the presence of dust in the large-scale host galaxy, whether diffuse or structured; 3) the evolutionary stage of the galaxy that hosts the quasar, including whether or not the galaxy is participating in a major merger, which can trigger the AGN but also can drive star formation and dust production. However, since the turn of the millenium and the advent of IR telescopes, obscured quasars have become understood to be a prominent component of the total quasar population (Zakamska et al. (2003), Glikman et al. (2004), Lacy et al. (2004), Lacy et al. (2007b), Treister et al. (2004), Lacy et al. (2013)) throughout cosmic time (i.e., Treister \& Urry (2006), Treister et al. (2009)), and as such, it is important for quasar studies to be designed to include them.

The concordance model of a $\Lambda \mathrm{CDM}$ universe is built around hierarchical structure formation. Hierarchical structure formation states that large masses are built from the merging of smaller masses, beginning with the formation of minute fluctuations or overdensities in the early universe, which are thereafter grown by gravitational effects (see, i.e., Eisenstein \& Hu (1999), Springel et al. (2005), and resources therein). Specifically this refers to dark matter haloes, where gravity dominates and no other feedback processes or interactions modify this underlying rule. Baryonic matter follows the dark matter, as it makes up only a fraction of the total mass available-but baryonic matter can cool and collapse to form dense structures, whereas dark matter is dissipationless. Baryonic matter can also experience feedback and forces other than 
gravity, and does, whether by radiation pressure, winds, jets, and outflows, or tidal shocking and other galactic harassment processes. The relationship therefore between the mass of dark matter haloes, the galaxy mass, and the mass of the supermassive black hole $(\mathrm{SMBH})$ formed within that galaxy is complex. This makes the study of environment, and its role in determining a galaxy's fate, difficult simply due to the number of variables involved.

One of the most powerful tools for exploring the richness of the large-scale environment of quasars is to explore their clustering. While quasars are rarely, if ever, found in actually virialized clusters, they have long been known to occupy overdense regions, with relatively high populations of galaxies around them (see, i.e., Bahcall (1969); Longair \& Seldner (1979); Yee \& Green (1984); Prestage \& Peacock (1988); Lacy (2000); Wold et al. (2000); Best et al. (2005); and Falder et al. (2011)). They can therefore be useful tracers of deep potential wells, and thus serve as a key for estimating the mass of the dark matter halo in which they and their associated galaxies are located (e.g. Hickox et al. (2011)). In work from the last twenty years, persistent evidence suggests that the halos in which quasars reside have a minimum mass throughout cosmic time, corresponding to $M>10^{12} M_{\odot}$ (Wake et al. (2004), Croom et al. (2005), Farrah et al. (2006), ?), Padmanabhan et al. (2009), Shen et al. (2009), Hickox et al. (2011)). According to dark matter halo models, the clustering strength of halos is expected to be weaker at higher z. Instead, observations find consistent clustering amplitudes throughout these different samples and studies. This implies an evolution instead of the linear bias - also known as the ratio of observed clustering to expected clustering, and a physical description of how baryonic matter responds differently from dark matter.

Environmental richness can be particularly useful for determining the origin sce- 
narios of different types of AGN. Since the exact mechanism that creates Type 1 and Type 2 quasars is not yet determined, this is especially useful. If the orientation model, which depends on a dusty nuclear component with a variety of possible opening angles, is the predominant cause for the differentiation between obscured and unobscured quasars, this would predict that since both types of quasars contain that component, they are essential identical. Therefore they would be expected to be found in similar environments. In contrast, according to the evolutionary model, if the dust obscuration is a temporary product of a recent burst of star formation driven by a major merger, the environments of obscured quasars are predicted to be denser, or demonstrate further evidence of increased merging likelihood. This latter prediction is limited somewhat in that the dust obscured quasars are expected to be relatively short lived before they blow out any circumnuclear material and become Type 1 objects - therefore if distinct environments are observed there is strong evidence for a merging scenario, but if the environments are found to be similar the merging scenario cannot be ruled out.

In this paper we present measurements of the quasar-galaxy cross-correlation function for a sample of obscured, unobscured, and reddened quasars, using data from the Spitzer Space Telescope. A description of the methods used is given in section 2.1; the samples used and their selection are described in 2.2. The methodology for these measurements is described in section 3 and the results are analyzed in section 4 . A cosmological model of $\Omega_{M}=0.3, \Omega_{\Lambda}=0.7$, and $H_{0}=70 \mathrm{~km} \mathrm{~s}^{-1} \mathrm{Mpc}^{-1}$ is assumed throughout the text. 


\subsection{Sample Selection}

\subsubsection{Quasar Selection and Obscured Quasar Identification}

Selected quasar data comes from the Spitzer Wide-area InfraRed Extragalactic (SWIRE) survey (see, e.g. Lonsdale et al. (2003)). This is a $49 \mathrm{deg}^{2}$ imaging survey taken in six fields at high galactic latitude, away from the Milky Way disk and as free as possible of interstellar and interplanetary dust contamination. The data were taken in the FIR with the MIPS camera and in the MIR with the IRAC camera; with IRAC, it reached a sensitivity of $\sim 5 \mu$ Jy for the 3.5 and $4.5 \mu \mathrm{m}$ bands and $\sim 50 \mu \mathrm{Jy}$ for the 5.8 and $8.0 \mu \mathrm{m}$ bands. MIPS sensitivity is a bit lower, but still more than adequate: $230 \mu \mathrm{Jy}$ at $24 \mu \mathrm{m}, 18 \mathrm{mJy}$ at $70 \mu \mathrm{m}$, and $150 \mathrm{mJy}$ at $160 \mu \mathrm{m}$.

The initial population and its detection is described in Lacy et al. (2007a) - 70 quasars that range in redshift from $z \sim 0.2$ to 4.5 . Of these objects, 25 are classified as Type 1/un-obscured quasars; 34 are Type 2/obscured quasars; and 11 are Type 1R (for reddened) quasars, which are somewhat between the two categories. The selection of possible AGN was based on the following color-selection in IRAC color-color space: $\log \left(S_{5.8} / S_{3.6}\right)>-0.1, \log \left(S_{8.0} / S_{4.5}\right)>-0.2$, and $\log \left(S_{8.0} / S_{4.5}\right) \leq 0.8 \log \left(S_{5.8} / S_{3.6}\right)$ +0.5 . Quasar type and redshift was determined with optical spectroscopy - Type 1 objects have broad lines and a blue continuum and the other earmarks of a standard quasar; Type 2 objects have high-ionization narrow emission lines that cannot be reproduced in a pure-starburst scenario; Type $1 \mathrm{R}$ objects have the broad lines of a quasar but no sign of a blue quasar continuum. See Lacy et al. (2004), Falder et al. (2011), and Lacy et al. (2013) for more detailed information on the methods of quasar identification and classification.

Since our choice of galaxy selection method (see next section) is most effective 
for removing lower-redshift contaminants and selecting high-z associated galaxies, we limit the quasar sample to the redshifts of $1.3<z<2.5$. This leaves 20 Type 1 quasars, 18 Type 2 quasars, and 7 Type 1R objects. After the initial data processing sorted galaxies by association with quasar by proximity within annuli from 0.1 to $9 \mathrm{Mpc}$, we found three underdense quasar objects (two Type 1s and a Type 2). After careful analysis, these three sources revealed contamination from bright stars within the $1 \mathrm{Mpc}$ annulus, producing a significant underdense population of associated galaxies. As a result, though we include the data in the data table, we remove these three outliers from the averages, the analysis, and our conclusions. It would be worthwhile to follow up these objects with a better mask for removing the stellar contamination, but that is beyond the scope of this paper.

\subsubsection{Galaxy Selection and Quasar Association Determina- tion}

The galaxy sample was obtained from the Spitzer Extragalactic Representative Volume Survey (SERVS) (see, e.g., Mauduit et al. (2012)). This is an $18 \operatorname{deg}^{2}$ survey to the depth of $23.1 \mathrm{AB}$ magnitude or $\sim 2 \mu$ Jy at 3.6 and 4.5 microns. It overlaps with five astronomical fields with complementary data and observations available (ELAISN1, ELAIS-S1, Lockman Hole, Chandra Deep Field South, XMM-LSS). SERVS is deep enough, and covers sufficient redshift, to offer a statistically significant number of galaxies to study at a variety of redshifts.

These galaxies are selected in the manner described in Falder et al. (2011): the 3.6-4.5 micron color of a standard galaxy SED varies in a predictable fashion with redshift, and a slice of color such that $3.6-4.5 \mu \mathrm{m}>-0.1$ selects galaxies that are at $z>1.3$. See Figure 3 in Falder et al. (2011). In more detail, the 3.6-4.5 $\mu \mathrm{m}$ color slice 
compares the slope between two portions of the spectrum of a galaxy, and at higher and higher redshifts the observed emission at 3.6 and $4.5 \mu \mathrm{m}$ is actually intercepting emission originally produced in the nearly optical regime. The slope described by the subtraction is more consistent in the optical as the varied impacts of dust and different emsision mechanisms play a very small role, hence at higher redshifts the measurement 3.6-4.5 $\mu \mathrm{ms}$ flattens out. Since the number of detected galaxies drops off significantly at higher redshifts, esp around $\mathrm{z} \sim 2.5$, this limits the redshift of the selected sample to $1.3<\mathrm{z}<2.5$

\subsection{Measuring Environmental Richness}

\subsubsection{Cross-correlation Functions}

The two-point correlation function is a measure of the clustering of objects around other objects in the sky, and an observable way to characterize the environmental richness. The cross-correlation between quasars and galaxies is necessary rather than the quasar autocorrelation function, due to the significantly greater number density of galaxies compared to quasars. Cross-correlation is preferrable to methods such as number-counting, the nth-nearest-neighbor distance, and Abell-type measurements, which are limited by the estimated magnitude of the quasar host rather than the magnitude-limit of the galaxy sample (Coil et al. (2007)). Much work has been done that uses the amplitude of the cross-correlation function to measure galaxy excess (i.e. Seldner \& Peebles (1978), Longair \& Seldner (1979), Prestage \& Peacock (1988), Wold et al. (2000), Wold et al. (2001), Coil et al. (2007), Padmanabhan

et al. (2009)), so we follow this method to compare our results with other work in the field. 
The angular cross-correlation function, $w(\theta)$, is defined as the probability that two objects that are separated by an angular distance of $\theta$ are found with a solid angle $\mathrm{d} \Omega$ on the sky:

$$
N(\theta) d \Omega=N_{g}(1+w(\theta)) d \Omega
$$

where $N_{g}$ is the average number of background galaxies per sterradian in the survey (and theoretically representing an average surface density of galaxies "in the field"), $N(\theta) d \Omega$ is the number of galaxies in $d \Omega$ an angular distance $\theta$ from the central object or source (in our case, the quasar). In general, $w(\theta)$ is assumed to take the form of a power law:

$$
w(\theta)=A_{g q} \theta^{1-\gamma}
$$

The amplitude $A_{g q}$ physically signifies the excess of galaxies associated with a quasar compared to random galaxy association in a field without quasars. This method is limited in that it is a measure only of visible association in $2 \mathrm{D}$ angular space, and not a realistic 3D spatial association. However it is possible to relate $A_{g q}$ to a measure of spatial association, $B_{g q}$ (again, see Longair \& Seldner (1979), Yee \& Green (1984), and Prestage \& Peacock (1988) for full details of the derivation), which is the amplitude of the spatial cross-correlation function, $\xi(r)$, which is defined similarly by

$$
n(r) d V=\rho_{g}(1+\xi(r)) d V
$$

where $\rho_{g}$ is the average number density of galaxies in the survey, also theoretically representing the field; $n(r) d V$ is the number of galaxies found in $d V$ at spatial distance 
$r$ from the central quasar. Similar to $w(\theta), \xi(r)$ has been assumed to take the form of:

$$
\xi(r)=B_{g q} r^{-\gamma}
$$

or

$$
\xi(r)=\left(\frac{r}{r_{o}}\right)^{-\gamma}
$$

and the amplitude $B_{g q}$ or scale length $r_{o}$ are also frequently reported means of determining environmental richness. Fortunately, $A_{g q}$ can be directly measured from annuli around the quasar, assuming an accurate background galaxy measurement, and can be used to derive $B_{g q}$ :

$$
A_{g q}=\frac{I_{\gamma}}{N_{g}} B_{g q} \Phi\left(m_{o}, z\right)\left(\frac{D}{1+z}\right)^{3-\gamma}
$$

where $\Phi\left(m_{o}, z\right)$ is a luminosity function defined by the limiting magnitude of the survey of galaxies and corrects for the number of galaxies expected within the given volume at the redshift of the quasar compared to how many were actually detected; $D$ is the comoving distance to the source and $I_{\gamma}$ is a definite integral that falls out of Longair \& Seldner (1979) and has, if we assume $\gamma=1.77$, a value of 3.78. Unless we encounter good reason to do otherwise, we will also assume spherical symmetry. For the luminosity function we will take the standard Schechter form,

$$
\Phi(M) d M=\left(0.4 \ln (10) \Phi_{o}\right) 10^{0.4\left(M_{\star}-M\right)(1+\alpha)} e^{\left(-10^{0.4\left(M_{\star}-M\right)}\right)}
$$

where $M_{\star}$ is the characteristic magnitude of the knee of the luminosity function, where the dominance of the exponential and power-law components switches; $\alpha$ is the slope of the power law; and $\Phi_{o}$ is a normalization factor, galaxies per $\mathrm{Mpc}^{3}$, 
that determines the total number of expected galaxies of the population - we use the total detected galaxies in the survey per unit volume. $M$, the magnitude, can be represented in terms of the distance, the limiting magnitude of the survey $m_{o}$, and the K-correction, $K_{z}$, which addresses the distinction between the emitted and observed light of a galaxy: $M=m_{o}-25-5 \log (D)-K_{z}$.

The primary limitation in obtaining a true 3D, spatially-associated richness is obtaining accurate redshifts to determine which of the huge number (on order of $10^{5}$ ) of galaxies in the survey is actually close enough to be spatially associated with the quasar. Without an excess of telescope time for spectroscopic follow-up, this challenge is best addressed with a color selection (see, for example, Papovich (2008), Falder et al. (2011)) - models suggest a particular evolution of $3.6-4.5 \mu \mathrm{m}$ color for a variety of different galaxy types, and a selection such that $3.6-4.5 \mu \mathrm{m}>-0.1$ yields predominantly galaxies with $1.5<z<5$. We assume statistical deficiency alone is sufficient to give a reasonable high-z cutoff - that the odds of background line-of-sight galaxies contributing significantly to the clustering measure is low enough to safely disregard. See, for example, Figure 19 in Mauduit et al. (2012) for support of this assumption.

The secondary limitation is completeness of the sample of associated galaxies, especially for higher redshift quasars, where low-luminosity objects are essentially invisible and one must extrapolate from the luminosity function and low-z data.

For this paper, we assume the power law form of $w(\theta)$ as in Eq. 1 and use the following estimator to obtain $A_{g q}$ :

$$
A_{g q}=\frac{N_{t o t}-N_{b}}{N_{b}} \frac{3-\gamma}{2} \theta^{\gamma-1}
$$

which is also used by Wold et al. (2000) and Wold et al. (2001) and has the benefit 
of being directly measureable from galaxy counts. Here $N_{\text {tot }}$ refers to the number of galaxies found within a certain angle $\theta$ of a given quasar. For this paper we select $\theta$ to be the angle that corresponds to $1.0 \mathrm{Mpc}$ at the quasar's redshift. $N_{b}$ is the expected number of background galaxies found within a circle of similar radius. This gives a measure of excess over background and

\subsection{Results}

\subsubsection{Background Galaxy Counts}

Differentiating between background galaxies and galaxies actually associated with the cluster is, as we point out above, a key component of calculating environmental richness. We explore several methods that make up the extrema of possible options without allowing thousands of telescope hours for spectroscopic redshift confirmation of galaxy association. The first method is focused on the large scale background: a measure of the total number of galaxies detected in the entire survey over the total survey area. The problem with this method is that it does not allow an easy accounting of edge effects or non uniformities in the survey images. The second method, at the other end of the spectrum, is focused on the background of each individual quasar. $A_{g q}$ is derived from galaxy counts in annuli around each quasar; at some sufficiently large radius, by definition, the clustering of galaxies around the quasar drops off and these annuli contain predominantly background galaxies. This is described by the power-law of the angular cross-correlation function, and while we assume the power $\gamma=1.77$, other work $(?), ?), ?)$ ) has explored cross-correlation fitting and found $\gamma$ with similar power-law drop offs and that the value of clustering amplitude is relatively insensitive to the precise $\gamma$. Thus for each quasar we calculate 
$N_{b_{\text {local }}}$, the number of background galaxies per $\operatorname{arcmin}^{2}$ found in an annulus with an internal and external angular radius equivalent to comoving distances with $8 \mathrm{Mpc}$ and $9 \mathrm{Mpc}$, respectively.

In terms of large scale structure, however, the universe is not uniform on scales of $10 \mathrm{~s}$ of $\mathrm{Mpc}$ - homogeneity cannot be attributed to the universe until the scale size considered is $100 \mathrm{~s}$ of Mpc. So it is entirely possible for $N_{b_{l o c a l}}$ to already be measuring a region that is overdense (or underdense) compared to a true background or field galaxy count. A compromise can be achieved by averaging over $N_{b_{l o c a l}}$, assuming the survey from which the quasars come covers a sufficiently wide area of sky and the quasars studied are well distributed throughout the survey. This produces $N_{b_{\text {global }}}=\overline{N_{b_{\text {local }}}}$, a measurement that averages over the large scale structure. We use both $N_{b_{\text {global }}}$ and $N_{b_{l o c a l}}$ to derive two separate measurements of $\mathrm{A}_{g q}$, labeled $\mathrm{A}_{g q}$ local and $\mathrm{A}_{g q}$ global in the table, but focus on $\mathrm{A}_{g q}$ local for further calculations.

\subsubsection{Calculation of $A_{g q}$ and $B_{g q}$}

Using our estimator (see Eq. 4) provides a measure of $A_{g q}$ at at a given separation from a quasar. We select a radius of $\sim 1 \mathrm{Mpc}$ to maximize the distinction between cluster and field (i.e. Yee \& Green (1984)). Galaxies from the sample are sorted into annuli with outer radii corresponding to $0.1,0.25,0.5$, and 1-9 $\mathrm{Mpc}$ and then the counts within $1 \mathrm{Mpc}$ are summed and the expected contribution from background galaxies within the same surface area is subtracted. This value is then normalized with respect to the background galaxy count value (as described in the previous section), to produce a measure of excess relative to an average field. These values are presented in columns 4 and 8 of Table 1 .

The transformation to $B_{g q}$ or $r_{o}$ relies on an understanding of the luminosity 
function of galaxies at this redshift. For this large of a sample population of galaxies, without expecting an excess of spiral, irregular, or elliptical galaxies, a Schechter function is the most reasonable approach to the luminosity function. We turn to the work of Stefanon \& Marchesini (2013), which explores the properties of luminosity functions in the $J$ - and $H$-band out to the higher redshifts associated with the quasars in this sample. The $H$-band values of the luminosity function they explore are redshiftdependent, so we sort our quasars into medium $1.5<z<2.0$ and high $2.0<z<2.5$ samples (note that the sample in this paper does not extend to the higher redshifts, where Stefanon \& Marchesini (2013) demonstrates higher uncertainty in the $\alpha$ and $M_{*}$ fitting). $\mathrm{K}(\mathrm{z})$ corrections to the luminosity function are obtained from Bruzual \& Charlot (2003) templates of a 0.02 metallicity, single stellar population at 5 Gyr (the K-correction applied varies negligibly with the population age), giving $K=0.5$. The luminosity function is strongly dependent on the normalization factor, $\phi^{*}$, so an independent estimate is derived from the total observed galaxies in and volume of the SERVS survey, $\phi^{*}=8.8 * 10^{-4} \mathrm{Mpc}^{-3}$. 
Table 3.1. Data and Results

\begin{tabular}{|c|c|c|c|c|c|c|c|c|}
\hline Name & Type & redshift & $A_{g q}$ local & $\begin{array}{c}A_{g q} \text { global } \\
\operatorname{rad}^{0.77}\end{array}$ & $A_{g q}$ error & $B_{g q}$ local & $\begin{array}{c}B_{g q} \text { global } \\
\mathrm{Mpc}^{1.77}\end{array}$ & $\bar{B}_{g q} \operatorname{Err}$ \\
\hline $160758.13+545046.1$ & 1 & 1.337 & 0.000164 & $-9.10 \mathrm{E}-05$ & 0.000207 & 181 & -100 & 228 \\
\hline $021830.57-045622.9$ & 1 & 1.401 & -0.000890 & -0.000796 & 0.000182 & -1030 & -921 & 211 \\
\hline $105353.93+570942.6$ & 1 & 1.425 & -0.000304 & -0.000250 & 0.000200 & -359 & -295 & 237 \\
\hline 021813.92-045241.2 & 1 & 1.443 & -0.000470 & -0.000290 & 0.000200 & -559 & -345 & 238 \\
\hline $161211.07+541910.9$ & 1 & 1.537 & 0.000182 & 0.000148 & 0.000213 & 231 & 189 & 271 \\
\hline $105010.58+574112.3$ & 1 & 1.55 & -0.000172 & $2.41 \mathrm{E}-06$ & 0.000208 & -221 & 3.10 & 267 \\
\hline $003829.92-434454.1$ & 1 & 1.567 & $2.27 \mathrm{E}-05$ & $8.20 \mathrm{E}-05$ & 0.000211 & 29.7 & 107 & 276 \\
\hline $105241.93+570245.6$ & 1 & 1.57 & 0.000128 & $4.22 \mathrm{E}-05$ & 0.000210 & 167 & 55.1 & 274 \\
\hline $105447.29+581909.4$ & 1 & 1.654 & 0.00184 & 0.000360 & 0.000219 & 2550 & 501 & 305 \\
\hline $104919.50+572332.9$ & 1 & 1.665 & 0.000548 & 0.000480 & 0.000223 & 767 & 672 & 312 \\
\hline 021902.58-044628.4 & 1 & 1.697 & -0.000180 & $-6.39 \mathrm{E}-05$ & 0.000206 & -258 & -91.8 & 297 \\
\hline 022002.09-050102.3 & 1 & 1.78 & $4.06 \mathrm{E}-05$ & $8.21 \mathrm{E}-05$ & 0.000211 & 61.7 & 125 & 321 \\
\hline $105414.79+571242.1$ & 1 & 1.8 & 0.000319 & 0.000441 & 0.000222 & 493 & 682 & 343 \\
\hline $160856.74+540313.8$ & 1 & 1.92 & 0.000108 & 0.000123 & 0.000213 & 181 & 206 & 359 \\
\hline $161228.96+542831.5$ & 1 & 2.053 & 0.000315 & 0.000324 & 0.000221 & 583 & 600 & 408 \\
\hline 022431.58-052818.8 & 1 & 2.068 & 0.000828 & $-7.81 \mathrm{E}-05$ & 0.000208 & 1540 & -146 & 388 \\
\hline 021850.06-050954.2 & 1 & 2.088 & -0.000172 & $-7.82 \mathrm{E}-05$ & 0.000209 & -325 & -148 & 394 \\
\hline $161129.04+545055.7$ & 1 & 2.101 & -0.000175 & -0.000280 & 0.000202 & -332 & -533 & 385 \\
\hline $021827.21-045456.6$ & 1 & 2.2 & -0.00116 & -0.00105 & 0.000176 & -2360 & -2140 & 359. \\
\hline $160735.60+543533.8$ & 1 & 2.385 & 0.000303 & 0.000453 & 0.000228 & 702 & 1050 & 530 \\
\hline $105255.34+571950.4$ & $1 \mathrm{R}$ & 1.45 & $-9.12 \mathrm{E}-05$ & $4.23 \mathrm{E}-05$ & 0.000210 & -109 & 50.3 & 250 \\
\hline $105133.59+574332.3$ & $1 \mathrm{R}$ & 1.58 & 0.000115 & 0.000135 & 0.000213 & 152 & 178 & 280 \\
\hline $105434.81+572023.3$ & $1 \mathrm{R}$ & 1.745 & 0.000154 & 0.000109 & 0.000212 & 229 & 161 & 315 \\
\hline $105319.54+571245.9$ & $1 \mathrm{R}$ & 1.853 & -0.000345 & -0.000330 & 0.000199 & -554 & -531 & 319 \\
\hline 003529.33-433611.4 & $1 \mathrm{R}$ & 2.261 & 0.000198 & 0.000314 & 0.000223 & 422 & 671 & 475 \\
\hline $105135.06+574704.8$ & $1 \mathrm{R}$ & 2.362 & $-5.69 \mathrm{E}-05$ & 0.000193 & 0.000220 & -130 & 440 & 502 \\
\hline $105201.92+574051.5$ & $1 \mathrm{R}$ & 2.467 & 0.000542 & 0.000868 & 0.000241 & 1330 & 2130 & 592 \\
\hline 022003.58-045145.6 & 2 & 1.33 & $-9.61 \mathrm{E}-05$ & $8.25 \mathrm{E}-05$ & 0.000212 & -105 & 89.9 & 231 \\
\hline 033532.19-284801.1 & 2 & 1.36 & 0.00110 & 0.000202 & 0.000216 & 1240 & 227 & 242 \\
\hline $160734.22+544217.3$ & 2 & 1.4 & 0.000239 & 0.000202 & 0.000215 & 276 & 234 & 249 \\
\hline 022004.90-045515.9 & 2 & 1.47 & 0.000125 & 0.000188 & 0.000214 & 152 & 229 & 260 \\
\hline $161052.74+543740.4$ & 2 & 1.494 & 0.000153 & $8.21 \mathrm{E}-05$ & 0.000211 & 189 & 101 & 261 \\
\hline $105515.18+570606.9$ & 2 & 1.59 & 0.000118 & 0.000188 & 0.000214 & 158 & 250 & 285 \\
\hline $105215.65+572942.0$ & 2 & 1.74 & -0.000144 & $2.42 \mathrm{E}-06$ & 0.000209 & -213 & 3.59 & 310 \\
\hline 022024.08-045709.7 & 2 & 1.767 & $-4.41 \mathrm{E}-05$ & $4.23 \mathrm{E}-05$ & 0.000210 & -66.6 & 63.8 & 317 \\
\hline $161039.04+543737.9$ & 2 & 1.83 & -0.000257 & -0.000277 & 0.000200 & -407 & -438 & 317 \\
\hline $105531.78+572714.9$ & 2 & 1.883 & 0.000200 & 0.000322 & 0.000219 & 328 & 530 & 360 \\
\hline $160824.74+542030.7$ & 2 & 1.914 & $-3.11 \mathrm{E}-05$ & 0.000136 & 0.000214 & -51.9 & 227 & 357 \\
\hline $161004.14+541338.7$ & 2 & 1.915 & -0.000348 & -0.000144 & 0.000205 & -586 & -243 & 345 \\
\hline $161115.91+540927.0$ & 2 & 1.92 & -0.000194 & $-7.76 \mathrm{E}-05$ & 0.000207 & -327 & -131 & 349 \\
\hline $003357.32-433601.7$ & 2 & 1.932 & 0.001033 & -0.000211 & 0.000203 & 1750 & -358 & 344 \\
\hline $022002.57-044758.9$ & 2 & 1.96 & 0.000144 & 0.000310 & 0.000219 & 249 & 537 & 380 \\
\hline
\end{tabular}




\subsubsection{Results as a Function of Redshift and Type}

There is no apparent trend in clustering amplitude with redshift, with variation of $B_{g q}$ for each type falling predominantly within the standard deviation of the sample $(85 \%$ of Type 1s; $86 \%$ of Type 1Rs; $89 \%$ of Type 2s; note that Yee \& Green (1984) points out that significant variation within a sample is expected and clustering amplitude results should only be considered statistically)). Since the clustering amplitude is also a measure of environmental richness, and the richness of environment is assumed to correlate with the mass of the halo in which the objects are found, this finding indicates that each type of quasar has a consistent halo mass across redshifts. The results presented in Fig 3.1 are consistent with the results of Hickox et al. (2011), Farrah et al. (2006), Hopkins et al. (2008), Wold et al. (2000), Yee \& Green (1984). Since models of dark matter haloes find dark matter to be more weakly clustered at earlier times, but the results of this work and the others mentioned above finds little evidence of a weakening in clustering in baryonic matter, this provides support for the idea that the bias factor (a measure of baryonic clustering compared to the underlying dark matter) varies with redshift.

Like Farrah et al. (2006), we also compare our results by type as presented in Table 2. Although the smaller sample size requires us to be cautious in our conclusions, it is clear from this data that Type 1, Type 1R, and Type 2 quasars are all highly clustered objects, as galaxy-galaxy correlation measurements find $B_{g q}$ on order of

Table 3.1-Continued

\begin{tabular}{|c|c|c|c|c|c|c|c|c|}
\hline Name & Type & redshift & $A_{g q}$ local & $\begin{array}{c}A_{g q} \text { global } \\
\operatorname{rad}^{0.77}\end{array}$ & $A_{g q}$ error & $B_{g q}$ local & $\begin{array}{c}B_{g q} \text { global } \\
\mathrm{Mpc}^{1.77}\end{array}$ & $B_{g q}$ Error \\
\hline $161017.93+542332.1$ & 2 & 2.05 & -0.000213 & $-7.80 \mathrm{E}-05$ & 0.000208 & -391 & -143 & 383 \\
\hline $160913.28+542322.0$ & 2 & 2.1 & 0.000132 & 0.000218 & 0.000218 & 252 & 414 & 415 \\
\hline $105203.66+572707.0$ & 2 & 2.43 & $-6.52 \mathrm{E}-05$ & 0.000180 & 0.000221 & -156 & 432 & 528 \\
\hline
\end{tabular}



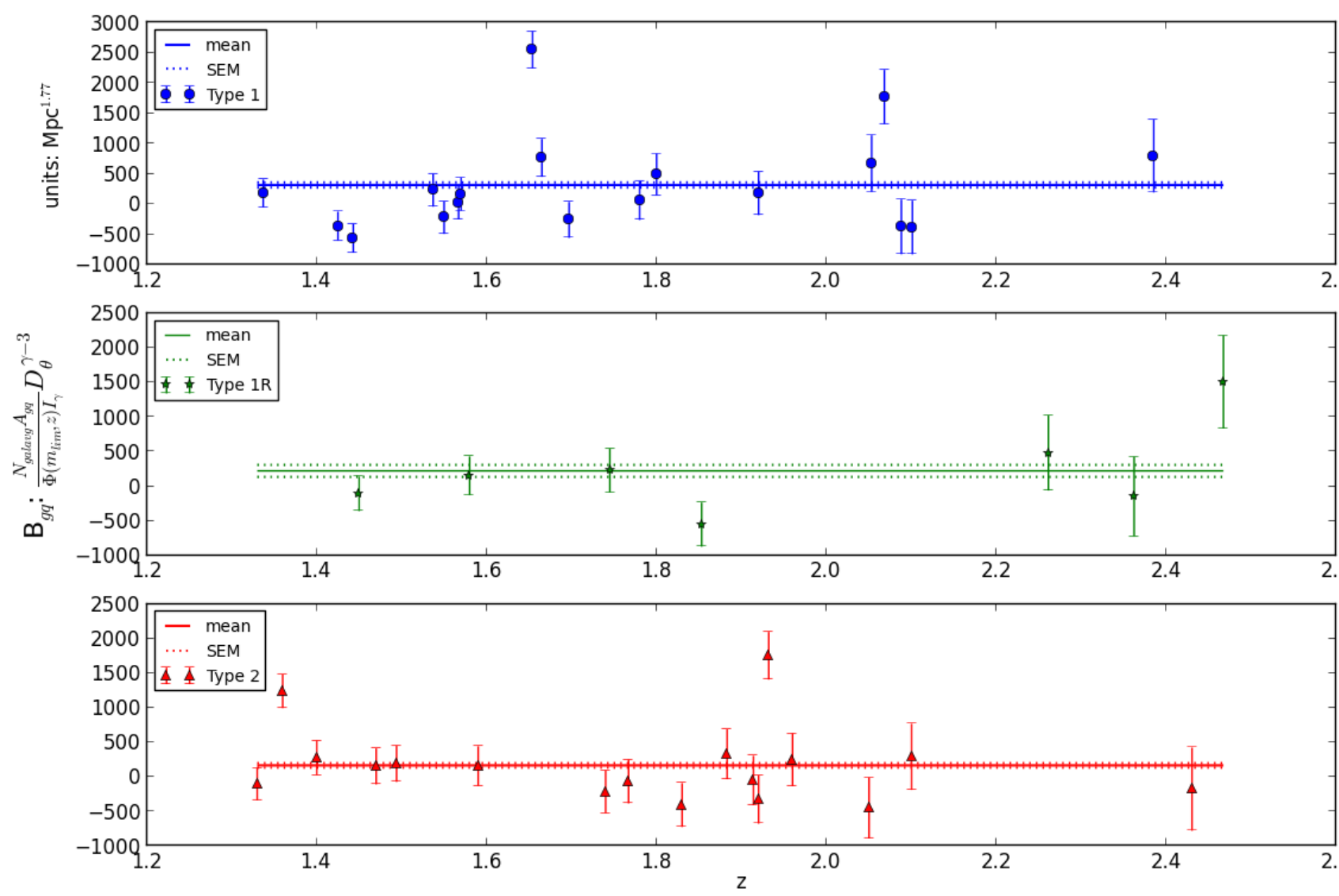

Fig. 3.1.- Comparing the local $B_{g q}$ values for all quasars in the sample. The solid lines indicate the mean of the sample, and the dashed lines are the mean \pm the standard error on the mean of each subsample. The given error bars on the points are error estimates based on the approximation of Yee \& López-Cruz (1999), using the number density properties of the entire galaxy sample.

Table 3.2. Average Angular and Spatial Covariance Amplitudes

\begin{tabular}{lcccccccc}
\hline \hline Type & $\mathrm{N}$ & $\begin{array}{c}\left\langle A_{g q}\right\rangle \\
\mathrm{rad}^{0.77}\end{array}$ & median $A_{g q}$ & $\begin{array}{c}S E_{\overline{A_{g q}}} \\
\left(\mathrm{Mpc}^{1.77}\right)\end{array}$ & $\begin{array}{c}\left\langle B_{g q}\right\rangle \\
\left(\mathrm{Mpc}^{1.77}\right)\end{array}$ & Median $B_{g q}$ & $S E_{\overline{B g q}}$ & $\overline{r_{o}}$ \\
$\mathrm{Mpc}$ & & & & & & & \\
\hline 1 & 18 & $1.847 \mathrm{E}-04$ & $1.176 \mathrm{E}-04$ & $2.800 \mathrm{E}-05$ & 319.6 & 173.7 & 42.91 & $26.0 \pm 8.36$ \\
$1 \mathrm{R}$ & 7 & $7.375 \mathrm{E}-05$ & $1.151 \mathrm{E}-04$ & $3.671 \mathrm{E}-05$ & 222.7 & 151.7 & 86.63 & $21.2 \pm 12.4$ \\
2 & 17 & $1.293 \mathrm{E}-04$ & $1.185 \mathrm{E}-04$ & $2.195 \mathrm{E}-05$ & 166.6 & 151.9 & 32.24 & $18.0 \pm 7.12$ \\
\hline
\end{tabular}


20.0. There appears to be no significant difference between the median values of each types. Type 1 quasars may demonstrate some evidence of higher clustering than their more obscured cousins, with a $\left\langle B_{g q}\right\rangle$ that is $\sim 2-3 \sigma$ higher than the mean of the Type $1 \mathrm{R}$ and Type 2 objects. However, this result must be treated with care, as a) it is revealed in the spatial clustering but indistinguishable in the angular clustering measurement, $A_{g q}$, indicating it may be an effect of the projection of $2 \mathrm{D}$ angular to 3D spatial correlation and the form of the luminosity function and b) as the medians of the sample are well within $1 \sigma$ of each other, this may be the result of a few over-dense objects but not an applicable conclusion to apply to the sample as a whole, especially considering the low size of the sample population. We perform a series of two-sample Kolmogrov-Smirnov tests on the three types, to determine if their underlying probability distributions are significantly different or if the samples can be expected to come from the same population. For all three combinations, the KS statistic is relatively high, $\gtrsim 0.17$, and the associated p-value $>0.75$. The values returned by this test are presented in Table 3. This indicates that the three different quasar types are all representative of the same underlying population, and supports an equivalent origin scenario for each type. Such results are consistent with an orientation-driven AGN model.

Table 3.3. Probability Test Results

\begin{tabular}{lcc}
\hline \hline & KS statistic & p-value \\
\hline Type 1 - Type 2 & 0.215 & 0.758 \\
Type 1 - Type 1R & 0.191 & 0.984 \\
Type 1R - Type 2 & 0.168 & 0.997 \\
\hline
\end{tabular}




\subsection{Conclusions}

\subsubsection{Comparison to Other Work in the Literature}

Our finding of an unevolving clustering amplitude from $1.3<z<2.5$ agrees with the work of, e.g., Wold et al. (2000), Myers et al. (2006), Myers et al. (2007), Coil et al. (2007), Gilli et al. (2009) and Ross et al. (2009), who also demonstrate a lack of evolution of clustering with redshift. This supports the concept that the linear bias between quasars and dark matter halos evolves with z, as suggested by Croom et al. (2004), Wake et al. (2004), Farrah et al. (2006), ?), Padmanabhan et al. (2009), Shen et al. (2009), Hickox et al. (2011), and numerous others. These findings do contrast with Shen et al. (2007), who find that the optically selected SDSS quasars of their high redshift $2.9<z<5.4$ sample have a higher correlation length at $z>3.5$, and Porciani et al. (2004), who similarly find a correlation length that increases with redshift for the 14000 quasars they investigate from the 2QZ survey. However it is important to note that both Shen et al. (2007) and Porciani et al. (2004) use high absolute magnitude selection criteria in the optical regime, increasing the likelihood of selecting the most luminous and most massive quasars - especially at higher redshifts, where the Malmquist bias can play a significant role. ?) actually point out that such a selection criteria may pre-dispose their sample to include particularly luminous objects, which have an increased likelihood to be found in densest, most clustered fields, whereas there is no other apparent trend in clustering at other luminosities (see, e.g. Croom et al. (2002), Adelberger \& Steidel (2005), Wake et al. (2005), Farrah et al. (2006), Myers et al. (2006), Serber et al. (2006), Myers et al. (2007), Shen et al. (2009), Thacker et al. (2009), and Donoso et al. (2014)). In addition both of these contrasting papers focus on optical identification of quasars, which does 
exclude the extensive obscured portion of the population; so overall the previous work of Porciani et al. (2004) and Shen et al. (2007) cannot be said to disagree with our own findings.

Overall, we find no significant difference between the Type 1, Type 1R, and Type 2 populations of quasars in SERVS in the redshift range $1.3<z<2.5$. Literature results as a function of color or obscuration are surprisingly diverse: Gilli et al. (2009), Shen et al. (2009), and Wylezalek et al. (2013), for example, find no dependence on color or obscuration for the objects in their samples, whereas Cappelluti et al. (2010) find their bluer objects to be more strongly clustered and Allevato et al. (2014) find their x-ray selected Type 1 AGN to be more strongly clustered. In contrast, Hickox et al. (2011) explore a matched sample of obscured and unobscured IR-selected AGN and find a $2 \sigma$ signifcant over-clustering of obscured Type 2 quasars compared to Type 1 objects. Similarly, DiPompeo et al. (2014) and Donoso et al. (2014) find that WISE-selected red/obscured quasars or AGN (respectively) are more densely clustered than their blue/unobscured counterparts. With the reasonable precision given in all of these papers and their varying sample sizes, the overall results of these types of studies may be more dependent on selection mechanisms than on color, and that while there is such extensive evidence for both similar and drastically different clustering environments for AGN with and without dust, it is not possible to distinguish a single dominant cause of the difference between the populations. It is likely that both orientation and evolutionary stage must play a role in how AGN present.

For future work it may be more valuable to explore clustering using selection mechanisms that incorporate quasars of diverse colors, but without the analysis focusing on obscuration as the predominant distinction. For example, a number of works (Wold et al. (2000), Mandelbaum et al. (2009), Falder et al. (2010), Donoso 
et al. (2010)) suggest a dependence of clustering strength on radio luminosity, and that that dependence may evolve with redshift - so a selection of IR or X-ray identified AGN explored through the lens of radio brightness may be a better probe of the relation between radio-source fueling and the halo-scale environment.

\subsubsection{Overall Findings}

In this paper we explore the redshift and color dependence of a sample of IR-selected quasars in the medium-high redshift regime. We report the following conclusions:

- No significant evolution of clustering amplitude with redshift is revealed, confirming the importance of a z-dependent linear bias as suggested in numerous other works.

- The Type 1, Type 1R, and Type 2 populations in this paper have relatively high inter-population KS statistic values, indicating that samples selected using the IR colors of Spitzer Space Telescope and the Lacy Wedge (Lacy et al. (2004)) are all derived from a similar overall population of quasars. This affirms the use of IR selection techniques as a means of producing a robust population that does not exclude the plentiful and under-explored obscured quasar population

- The clustering amplitudes and/or correlation lengths of each of the sample populations indicate that Type 1, Type 1R, and Type 2 quasars are comparably clustered, with little evidence of either obscured or unobscured quasars being more densely clustered at any redshift, especially accounting for one or two densely clustered outliers in the Type 1 population.

- Future work might focus on a) the difference in environments of Type $1 \mathrm{R}$ and

Type 2 objects; b) the relation of halo-scale environment to the mechanisms 
of radio-jet fueling; and c) the effect of a few extreme-density objects on the diverse results in the literature based on type.

- Further work for this paper will include bias factor and halo masses calculations, and exploring any trends with archival data available for these objects 


\section{Chapter 4}

\section{Conclusions and Future Work}

\subsection{Overall Conclusions of This Dissertation}

\subsubsection{Halo-scale Environments of Obscured and Normal Quasars at High Z}

The bulk of my dissertation research has focused on exploring the quintessential SMBH-dominated population, quasars. Found in a vast range of lookback times, quasars serve as a means to explore galaxy environments across cosmic history. In particular I have studied the large-scale environments of the fields of Spitzer Widearea IR Extragalactic (SWIRE) quasars from $1.3<z<2.5$ carefully selected to include obscured quasars which are frequently under-represented in AGN investigations due to their lack of optical brightness and subsequent difficulty in detection. Infra-red selection techniques using color-color diagrams from Spitzer data provide a means of distinguishing AGN that is relatively independent of obscuration by dust (Lacy et al. (2004); such selections can be further confirmed using optical spectroscopy.

One of the most powerful mechanisms for characterizing large-scale environments 
is the use of cross-correlation functions to explore the clustering of galaxies around the object of interest (in our case, quasars). The benefit of cross-correlation is that there is no need for a large sample population for the objects of interest; instead the statistical rigor depends on the objects with which they are crossed, in this case galaxies which are many thousands of times more common throughout the universe. It is the properties of the galaxy sample (from the Spitzer Extragalactic Representative Volume Survey, SERVS: an $18 \mathrm{deg}^{2}$ survey that extends to $\sim 2 \mu \mathrm{Jy}$ at 3.6 and 4.5 $\mu \mathrm{m})$ that determines the limits of the cross-correlation: we narrow our selection of quasars to 45 objects that can be matched to the high redshift range of SERVS galaxies. With the clustering estimator $A_{g q}$ as described in Wold et al. (2000), we derive median correlation lengths of $r_{o}=18.4 \pm 8.36,17.1 \pm 12.4$, and $17.1 \pm 7.12$ Mpc for the Type 1, Type 1R, and Type 2 quasar populations, respectively; the mean correlation lengths are more diverse, giving $\left\langle r_{o}\right\rangle=26.0 \pm 8.36,21.2 \pm 12.4$, and 18.0 $\pm 7.12 \mathrm{Mpc}$ for the same types. The difference between the two measures is apparent in Figure 1 of Chapter 3: several of the Type 1 quasars are significantly overdense.

The clustering analysis in this dissertation reveals two key findings. First, we find no evidence for evolution of clustering amplitude with redshift. The amplitudes of our clustering analysis are even on par with the findings of Wold et al. (2000) and Wold et al. (2001), from $0.5<z<0.8$ samples of radio loud and quiet quasars. This confirms previous work that suggests a minimum halo mass is necessary to form supermassive black holes and other extremely luminous and massive objects such as ULIRGS, and that the linear bias or ratio of the mass of SMBH-containing halos (from observations) to the mass of dark-matter halos (predicted by theory) instead evolves with redshift.

Secondly, using the median values of our samples, we conclude that there is no dif- 
ference in clustering amplitude based on quasar type or obscuration, although there is some evidence that particularly bright objects may be found in particularly overdense fields (the so-called "extrema effect" that is evident in numerous other works in the literature). This both conflicts and coincides with different aspects of the literature, and while some of the differing conclusions can be attributed to the extrema effect or selection effects, there is yet no clear consensus as to whether obscured quasars exist or form in different environments than normal quasars. However, the results of this work are consistent with either the obscured and unobscured objects being the same objects viewed at different orientations, or, in the evolutionary hypothesis, with a rapid $\left(\leq 10^{8} \mathrm{yr}\right)$ evolution of AGN from an obscured to an unobscured state, and are inconsistent with a substantially different environmental triggering or feeding mechanism for the obscured and unobscured populations.

\subsubsection{Circumnuclear Environments and the Presence of AGN in LIRGs}

In addition to my work on the halo-scale environments of quasars, I also investigated the smaller-scale circumnuclear environment of Luminous Infrared Galaxies. Using high-resolution mid-infrared imaging, I characterized the morphology and brightness surface density on the $\sim 100$ pc scale within the central 1-2 kpc of ESO 420-G013, NGC 2369, NGC 4418, NGC 5135, and MCG-03-34-064. The 12.81 and $13.01 \mu \mathrm{m}$ images of these objects reveal varied morphological structure with knots of star formation, as well as two compact sources that are likely unresolved and show evidence of containing an AGN. Circumnuclear flux densities for most of the sample are on par with the total galaxy $12 \mu \mathrm{m}$ flux densities, which puts a lower limit on brightness surface densities for four of the objects at $10^{10} L_{\odot} k p c^{-2}$. 
From this work it is clear that the circumnuclear regions of LIRGs are particularly important in determining their characteristics. The high brightness surface densities demonstrate that compact structures can produce significant amounts of power within the central 1-2 kpc. We can extrapolate similarly that large amounts of star formation are occuring on the same scale of $\sim 100$ pc. Such compact luminosity, and the star formation rates it indicates, reveals the role that the circumnuclear region plays in the evolution of a galaxy. Star formation consumes available gas that might otherwises fuel the AGN. It can produce winds and outflows that can drive further gaseous material out of the circumnuclear region entirely, slowing or halting the fueling of the $\mathrm{SMBH}$, and driving changes in the accretion efficiency. It may also serve as a mechanism for stripping gas of angular momentum, or through the random orientation of star forming regions and their contents, actually drive gas further into the nuclear region.

The diverse morphological structure confirms the importance of the circumnuclear environment. The presence of a circumnuclear spiral in one object (as well as the possibility of circumnuclear rings in two other objects) suggests that not only can the contents of the circumnuclear play a perhaps meager random role in the feeding or quenching of a supermassive black hole, but that they can serve as one more crucial step among many nested steps in the stripping of mangular momentum from gas that is essential for powering an AGN. This important feedback process (of the circumnuclear environment on the SMBH-scale environment) suggests that AGN triggering can occur in bursts throughout the history of a galaxy as long as it has gas that can enter the circumnuclear region - that an AGN need not be "on" continuously, but that after it eventually ceases activity it may again be turned on at any point based on the effects of the circumnuclear region and the host galaxy, on longer time scales 
than week-year variability observed for the most resolved nuclear emission and on shorter time scales than the merging time. In such an interpretation, the compact source objects (NGC 4418 and MCG-03-34-064), with signs of an AGN within their point-like source, may represent a further nested step of driving gas onto the accretion disk. However this would require significantly higher resolution imaging to determine - 0.1 - 10 pc scale - and that is beyond the ability of available MIR telescopes.

\subsection{Future Work}

\subsubsection{Approaching a True Understanding of AGN and the Role of Sample Selection}

If there is one message at the core of this dissertation, it is that the study of active galactic nuclei is a complex, cutting-edge field that involves a huge range of luminosities, spectral characteristics, masses, size scales, and timescales, and that observational limits further cloud the objective of exploring true properties of AGN. From the work in Chapter 2, we see that improving the imaging resolution by an order of magnitude is not necessarily sufficient to distinguish the relative roles of two different luminosity sources, stars and AGN, as well as that the intermediate scale (the circumnuclear region, between the host galaxy and the proper nucleus) can still have an influence on both the larger and smaller scale environments. From the work in Chapter 3, we see that the large, halo-scale environment, while widely variable on an individual level, is a necessary component for creating the most luminous and massive of SMBH objects. Both of these research projects would benefit from cross-over: the large-scale environment of LIRGs is relatively unexplored, as are the properties of galaxies that host quasars, especially at high redshift. 
The future of understanding AGN relies on defining populations less by observational limits than by intrinsic AGN or environmental properties. For example, the color-color cuts of the Lacy Wedge described in Chapter 3 select objects based on the relative strength of star formation to AGN within the MIR regime-this is why the selection mechanism finds both obscured and unobscured objects.

For my future work, there are three projects I propose that relate to this idea of precise population definition. Two extend the work on the quasar sample from Chapter 3. First, I propose to investigate the dependence of the spectral energy distributions of these objects, revealed by extensive archival data from the X-ray to the radio, on the host-galaxy environment. The quintessential quasar SED is broad across the full spectrum but there has been little attempt to explore how the structure of the SED might correlate with halo-scale environmental properties. Secondly, I propose to explore the medium/small scale environment of these quasars by observing the host galaxies in which they are found using high-resolution Hubble Space Telescope imaging (see Appendix A for the HST proposal). The second relies on defining a new sample population of AGN (Feedback AGN) based on whether or not the AGN shows direct signs of interacting with its host galaxy. This relies on observations with the Arecibo Observatory (see Appendix B for the Arecibo Observatory proposal). 


\section{Appendix A Exploring the Medium-scale Environ- ment of Host Galaxies with the Hub- ble Space Telescope}

\section{Appendix A.1 Scientific Justification}

Quasars are among the most luminous objects in the universe, allowing them to be viewed at a huge range in redshift. They are believed to be part of the evolutionary sequence through which most massive galaxies pass (i.e., Sanders et al. (1988a), Sanders et al. (1988b), Hopkins et al. (2008)). This belief derives from several key characteristics of quasars. First, their intense luminosity, which is attributed to an active galactic nuclei (AGN), can be powered by gas-rich mergers, which remove angular momentum from gas and provide fuel to the nuclear region (see, e.g., Silk \& Rees (1998), Hopkins et al. (2006)). Secondly, the observed correlation between black hole mass and bulge mass suggests the co-evolution of the black hole and the galaxy in which it is embedded (e.g., Ferrarese \& Merritt (2000), Gebhardt et al. (2000), Peterson (2008)). Finally, some observations of host galaxies suggest that they tend to be significantly disturbed systems (i.e. Sanders \& Mirabel (1996), Bennert et al. (2008), Urrutia et al. (2008)).

Further studies (e.g., Treister et al. (2012)) indicate that secular processes can play a role in fueling quasars and other Active Galactic Nuclei (AGN), especially at low luminosities, but that mergers become more important as the luminosity of the object under consideration increases. However, this luminosity dependence has recently been called into question by Villforth et al. (2014), who find no trend for more luminous AGN to be more likely to be in morphologically-disturbed systems. 
To further confound the question, these studies used samples of AGN selected in a wide variety of different ways: in particular, the X-ray selection used by Villforth et al. (2014) may bias against gas-rich hosts that would be the typical results of "wet", i.e. gas-rich mergers. We thus wish to investigate whether or not such a trend of morphology with luminosity exists by studying luminous quasars from a sample selected in the mid-infrared, which should be almost unbiased with respect to dust and gas content of the host galaxy.

In the past ten years it has been increasingly obvious that there exists a distinct portion of the quasar population that has been relatively ignored due to selection effects. Dust-obscured quasars (henceforth obscured or Type 2 quasars) have been detected and confirmed through many methods and in many surveys (Cutri et al. (2002), Gregg et al. (2002), Zakamska et al. (2003), Lacy et al. (2004), Stern et al. (2005), Donley et al. (2005), Lacy et al. (2007a), Lacy et al. (2013)). In particular, an increasing number of studies (Martínez-Sansigre et al. (2005), Lacy et al. (2007b), Reyes et al. (2008)) indicate that not only are these obscured quasars a non-trivial portion of the population, but that they indeed may contribute equally or even dominate the quasar population by number, making the conclusions and inferences drawn about quasars, their hosts, and their role in galaxy evolution incompletely informed. In order to complete our understanding of quasars and their role in the grand scheme of the universe, a thorough exploration of this understudied, dust-obscured population of quasars is necessary.

As part of a broader thesis studying the nature and environments of obscured quasars, we propose to use the Hubble Space Telescope's unique sensitivity and angular resolution to observe and characterize the host galaxies of a sample of 68 midinfrared selected quasars, mostly obscured, but including about $30 \%$ of normal, blue 
quasars. We propose to use both the optical and the NIR filters on the WFC3 camera in order to explore the distribution of dust and star formation throughout the host galaxies.

In order to determine the degree of morphological disturbance in our host galaxies we can use a number of objective measures of morphology. We will follow Urrutia et al. (2008) and plot concentration index against the Gini coefficient; such a plot allows objects with extended low surface brightness structures such as tidal tails to stand out. In the cases where our hosts are of regular enough morphology, we will fit Sersic models and subtract them to reveal morphological signs of interaction (i.e. Bennert et al. (2008)). In addition to the above measures we will also map the ratio of optical to NIR light to map the distribution of dust in our host galaxies.

Essential to probing these characteristics are high resolution and high sensitivity observations. Since quasars are not found in the local universe, a high angular resolution in combination with sufficient sensitivity to get good surface brightnesses is necessary to look for signs of disturbance to determine the proportion of interacting hosts. In addition to its unmatchable resolution, the multi-wavelength capabilities of the HST are also essential for such detailed study. The near-IR will allow us to probe obscured emission with a resolution and surface brightness sensitivity that will provide information on tidal features and multiple nuclei that would be missed by conventional ground-based near-IR imaging. Simultaneously, the optical allows identification of recent star formation and identifying the distribution of dust obscuration. Only nine of our targets have suitable guide stars for adaptive optics observations in the near-IR; in these cases we will only take HST images in the optical, shortening the snapshots for those targets, as there is no sufficiently high resolution ground-based optical system. 
We have thus selected a sample of quasars to investigate that a) represent a range of quasar types; b) are distributed over a large portion of the sky; and c) are at redshifts that make host galaxy observations feasible. Selection was made by matching the SDSS data in the deep Stripe 82 (a $250 \mathrm{deg}^{2}$ equatorial stripe observed multiple times by SDSS) with the Wide-field Infrared Survey Explorer (WISE) catalog and selecting likely candidates for quasars based on mid-infrared colors (see, e.g., Lacy et al. (2007a) for mid-infrared color selection techniques and Lacy et al. (2013) for spectroscopic confirmation techniques). Some of these are already classified as quasars by the SDSS, and others have spectra as part of the SDSS galaxy survey. About 25\% have no SDSS spectroscopy, and we used the Lick 3m telescope to obtain spectra for these objects. We now have a sample of 68 objects with $z>0.25$ and 22 micron fluxes $S_{22}>20$ mJy (ensuring bolometric luminosities $L_{b o l} \geq 10^{12} L_{\odot}$ so that these objects are in the quasar regime). To give an idea of what the Type 2 quasar host galaxies might look like, see Fig. 1; these are the only 6 mid-infrared selected quasars that have been observed with HST before. The nature of the selection ensures that we will concentrate on the less well-studied obscured quasars, which also have the significant practical advantage of lacking a bright nucleus that would require accurate PSF subtraction; however several Type 1 quasars are also in the sample, and will allow us to make comparisons to the unobscured population.

Once our targets have been observed, we will be able to compare with HST images of lower luminosity AGN at similar redshifts from CANDELS and COSMOS. This will allow us to search directly for evidence of a change in the triggering and fueling mode of AGN as a function of luminosity. 
Fig. 4.1. - HST images of the only six Type 2 mid-infrared selected quasars yet observed with this high resolution. Host galaxy substructure is clearly resolved. Longwavelength images were colored red, and the short-wavelength images were in green. Images originally published in Lacy et al. (2007b).
a) SSTXFLS J171831.5595317
b) SSTXFLS
J172123.1601214
c) SSTXFLS J172458.3591545
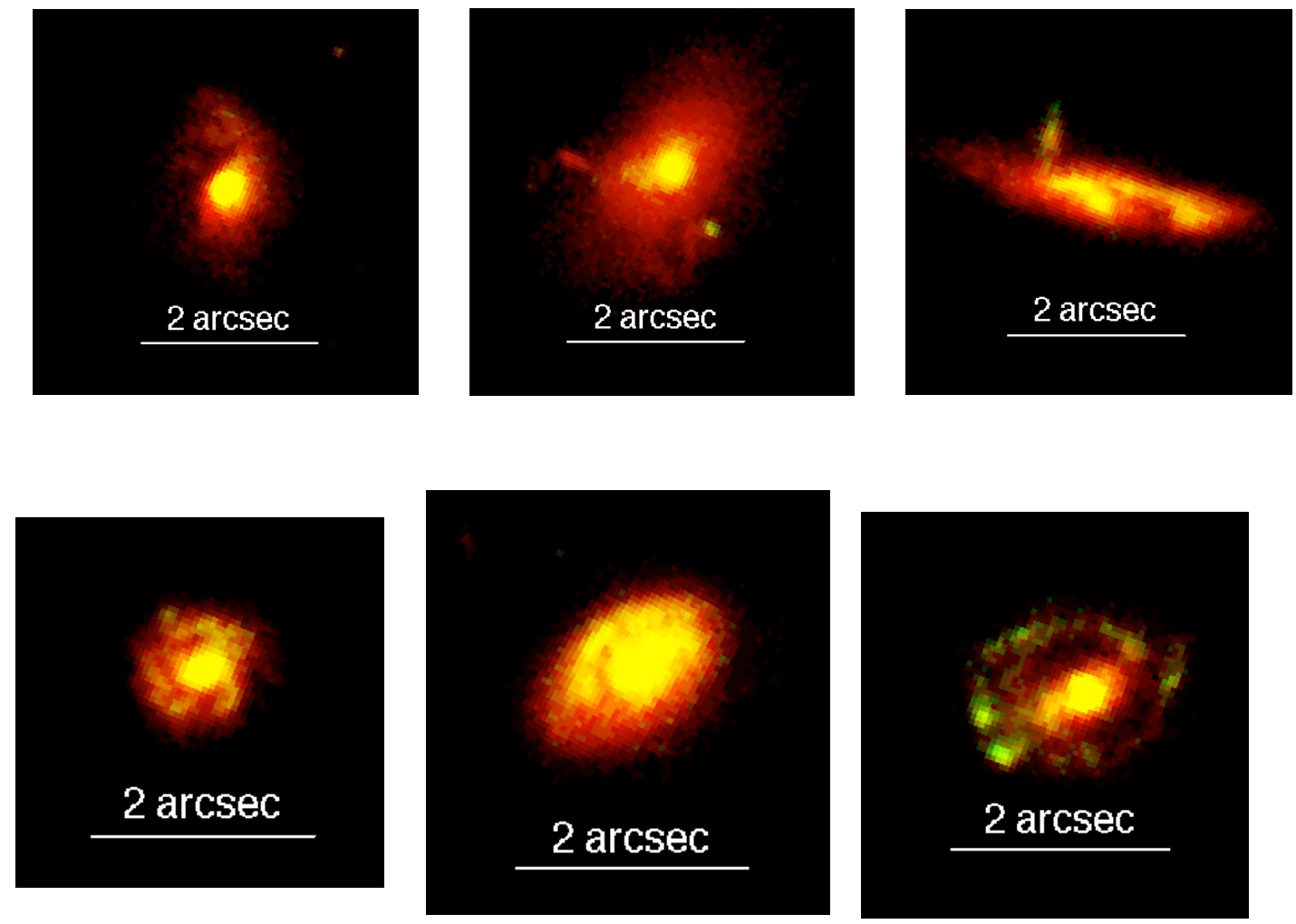


\section{Appendix A.2 Describe Observations}

We will observe our targets in snapshot mode using WFC3. One observation will be made in the near-infrared F160W filter, and one in an optical filter below the 4000 $\AA /$ Balmer break: F438W for objects with $0.25<z<0.5$, or F475W for $0.5<z<1$. This selection also avoids strong emission lines such as [O II] 3727 and [O III] 5007. In order to estimate the signal-to-noise required, we use the example of a type- 2 quasar (a type-1 object should have a similarly-bright host galaxy). Our objects have $0.25<z<1$, with a median redshift $\approx 0.5$, and optical magnitudes in the range $g \approx 17.5$ for objects at $z \approx 0.3$ to $g \approx 21.5$ for objects at $z \approx 1$. Expected H-band magnitudes lie in the range $16<H<19$, based on our attempted observations of quasar hosts at similar redshifts with adaptive optics (Lacy et al. (2002)) and detections or limits from 2MASS.

We assume that the host galaxies have a half-light radius of $3 \mathrm{kpc}(0.8-0.4$ arcsec over the $z=0.25-1.0$ redshift range); this leads to mean surface brightnesses within the half-light radii of $A B=18.2-21$ magnitudes per square arcsecond in g-band (assuming the fainter objects are at higher redshifts). The lowest surface brightness objects will be detected through the F438W filter at 9-sigma in a $3 \times 3$ pixel box in $2 \times 300$ s exposures (assuming the NGC 1068 SED and a 12 electron post-flash), or at 19-sigma through the F475W filter. This will give us plenty of signal-to-noise to fit a profile, and also enough depth to find faint features at much lower isophotes. In the near-infrared, our corresponding surface brightnesses at the half-light radii will be $H=16.8-18.3$ Vega magnitudes per square arcsecond. We will be attempting to search for faint tidal features at isophotes about 10-times lower, and will use F160W and integrate for $2 \times 180$ s to detect these features at 12-sigma in $2 \times 2$ pixel boxes.

For the Type 1 objects we will attempt a PSF subtraction. As these are snapshots 
the PSF will be slightly degraded compared to normal observations, but we expect to find enough stars on the frame to measure the degradation, and we can also use archival observations to determine a PSF that is adequate for our purposes. The high surface brightness sensitivity of WFC3 will allow us to trace the host galaxy in these

relatively low redshift quasars far beyond the region affected by the PSF, so a high fidelity PSF will not be required.

\section{Appendix A.3 Duplications}

Of our initial sample of 69 quasars, only two objects have previously completed HST observations: J205601.68-001613.1, which was observed with the F606W filter in the optical on ACS, and J030000.56+004827.9, which has both NIR and optical observations. We exclude J030000.56+004827.9 from our sample, but keep J205601.68001613.1 as the comparison between the optical and NIR bands is essential for our research goals and it has not been observed in our selected filters. 


\section{Appendix A Targetting Feedback in AGN with the Arecibo Observatory}

\section{Appendix A.1 Scientific Justification}

Orientation (i.e. Antonucci (1993), Urry \& Padovani (1995)) with respect to a dusty toroid, and evolutionary stage (i.e. Sanders (1998), Hopkins et al. (2008)) of the host galaxy remain the basic models for approaching an understanding of active galactic nuclei. But more recent data adds layers of complications to these simple explanations. Among the relevant data is the detection of intermediate mass black holes of respectable sizes within dwarf galaxies; the extensive presence of significant star formation (and to a lesser extent other secular processes) in even the most massive and luminous galaxies; the varied environments in which AGN of the same types are found; and the detection of greater numbers of Compton-thick, optically obscured, or dusty red quasars at a variety of redshifts, evolutionary stages, and environments.

It is therefore necessary to explore supermassive black holes (SMBH) from different lenses than those constructed by evolution and orientation. The lens we are considering is the interaction between the AGN and its host galaxy. From an orientation perspective, such a process is governed by the distribution of dust and gas in the circumnuclear and nuclear regions; from an evolutionary perspective, it is driven by the large-scale environment and interaction with neighboring galaxies. We seek to explore a sample not selected by gas distribution or large-scale environment, but by its level of feedback, as a more intrinsic property of these sources.

We seek to study the interaction of the AGN with the ISM of its host galaxy by observing neutral HI gas in the host and how it is driven by outflows and jets from the AGN. In the study of galaxies and their evolution, gas outflows play a significant role 
in returning gas to the host from the nuclear region as well as providing a means of both heating and efficient cooling depending on the mechanism. Such recycled gas can be enriched (and often is, in the case of starbursts, superbubbles, and supernovae), but neutral gas can also be moved around by jets, diffused, and re-condensed into cold clouds. In particular, HI has been detected in absorption against bright radio sources in the past: quintessentially, Morganti et al. (2003) detected a $1400 \mathrm{~km} / \mathrm{s}$ primarily blue-shifted HI absorption signature in 3C 293 attributed to an outflow interacting with the ISM. Since then numerous detections have been made of broad HI absorption lines stemming from outflows: Gupta et al. (2005), Morganti et al. (2005), Oosterloo \& Morganti (2005), Croft et al. (2006), Gupta et al. (2006), Gupta \& Saikia (2006), Kanekar \& Chengalur (2008), Wild et al. (2008), Morganti et al. (2009), Allison et al. (2012), Chandola et al. (2012), Teng et al. (2013), to name a few.

More specifically, we propose a two-stage project - to first observe a sample of local quasars for broad HI absorption as a signature of jet interaction with the host galaxy, and then to follow up those objects that have associated HI absorption with VLBI observations to directly characterize the gas distribution and outflows present within the circumnuclear or nuclear regions of these galaxies. Such feedback is a direct signature of an interaction between the SMBH and its host galaxy, and using VLBI to trace that signature will provide morphological information about the host galaxy ISM. In addition, since the source of such feedback is a jet, VLBI observations of the nuclear region at several frequencies would allow us to map the radio emission and the spectral index within the region most closely associated with the AGN.

HI absorption associated with a given radio galaxy is notoriously diffuse, with low optical depths on the order of 0.001 , and broad: typical lines are on the order of $1000 \mathrm{~s}$ of $\mathrm{km} / \mathrm{s}$. This makes the use of the Arecibo Observatory telescope essential for this 
proposal: first, for its sensitivity and broad bandwidth L-band receiver for initially detection HI absorption lines among the sample; and second, for the sensitivity it will contribute to the VLBI observations, allowing more time to be spent on resolving the nuclear region at multiple wavelengths and thus crafting a more complete picture of the physical structures and feedback processes contained within this region.

\section{Appendix A.2 Sample Selection}

Traditional radio-loud galaxies are defined by having $5 \mathrm{GHz}$ flux and B-band flux

ratios of $\frac{F_{5}}{F_{B}}>10$. This automatically creates a dependence on the optical characteristics of the galaxy. We propose therefore to select a sample from the FIRST Bright Quasar Survey (FBQS), which encompasses radio-loud, radio-intermediate, and radio-quiet sources and extends beyond $\mathrm{z} \sim 3$. Constraining the DEC of the objects to a range of + 0:0:0 to $+35: 0: 0$ to make sure that there is sufficient tracking time available to follow-up these objects with VLBI using Arecibo, the FBQS gives $\sim 60$ non-stellar objects of a variety of redshifts, optical rednesses, and $\frac{F_{5}}{F_{B}}$ ratios. These span the RA of $07 \mathrm{~h}$ to $17 \mathrm{~h}$, meaning they can be observed throughout the year or during the day if the sun is not within the beam (and the local daytime RFI is not significantly stronger, although the breadth of the absorption line should make it observable despite narrow RFI).

\section{Appendix A.3 Phase I: HI Absorption Line Detection}

The main concern in detecting the broad absorption lines that signal the presence of nuclear or circumnuclear jets and AGN feedback is the redshift of the source. Arecibo's L-band receiver, which spans 1.15 to $1.73 \mathrm{GHz}$, will be able to detect these lines only out to a redshift of $\mathrm{z} \sim 0.2$; this cuts the sample size down to 15 objects 
but is convenient for high-resolution follow-up imaging. This localized sample of 15 objects will form the initial experiment. At higher redshifts, the $430 \mathrm{MHz}$ and 327 $\mathrm{MHz}$ receivers are sufficient to detect objects at $z>2.2$; this is only three objects, but could serve as a proof-of-concept for future work. With the VLA Sky Survey and other upcoming deep radio surveys (GMRT), there will be an increase in the high-z radio objects available to sample, and this useful Arecibo-based observation plan will serve as a means for exploring feedback and AGN/host interactions as a function of redshift.

Although the absorption peak of HI is typically quite low, at order of $10 \mathrm{~s}$ of $\mu \mathrm{Jy}$, the peak does not need to be reached to establish a detection. Rather, the breadth of the absorption line serves as a signature of HI closely associated with the quasar, driven by a strong outflow. With that in mind, a typical integrated flux for the local sample is on order of $1 \mathrm{mJy}$ and we seek line detection down to at least $0.05 \mathrm{mJy}$ to be sure of the breadth of the absorption. Although this may vary given the exact strength of the source, observations on the order of 20-30 min are expected to be sufficient for these objects. That yields a total requested observing time of 5-8 hours initially, easily accomplished within the course of one observing semester. Even if some of the fainter objects require a full hour of observation, 15 hours over the course of the semester including daytime observations remains feasible.

\section{Appendix A.4 Phase II: VLBI Multi-band Follow-up and Nu- clear Jets}

The second stage of this project relies on high-resolution observations, and separate VLBA/HSA proposals are being developed to support this further work. Previously, Oosterloo (2004) and Morganti et al. (2005) demonstrate that identifying the location 
of the outflowing HI gas is vital for understanding its origin: Oosterloo (2004) suggest that the rapidly moving HI clouds are accelerated by shocks from the jet interacting with the ISM. Such a mechanism could directly trigger star formation in the host galaxy. VLBI observations of the distribution of HI in objects with outflows thus provides 1) the scale size of the jet and thus a timescale for AGN/host feedback; and 2) the location and distribution of HI outflows and thus a characterization of the type of feedback or interaction.

In addition to HI-centric observations, it is also feasible to extend the VLBI observations to several different wavelength bands. This provides a means for a third scientific exploration: 3) mapping of the spectral index of radio emission within the resolved region, which creates a means to distinguish between different dominant power sources and perhaps to disentangle the AGN and star-formation contributions of each object (with a spectral index of $\alpha \sim 0.7$ indicating the traditional synchrotron emission of the AGN).

An estimate of time necessary for Phase II depends on the number of broad HI absorption systems observed during Phase I and their redshift (which will affect what spatial resolution is required, and thus the expected FOV necessary to observe the circumnuclear and nuclear regions. This will place a limit on the number of channels we can use for spectral resolution to prevent bandwidth smearing, and thus our sensitivity estimation depends on how many and which channels we could use). However, note that the initial sample was selected to have a DEC range that will allow nearly an hour of tracking time with Arecibo to maximize the number of available baselines with which the objects could be observed and thus the resolution scale available for the observations. 


\section{Appendix A.5 Conclusion}

It is important to note that even simply detecting the broad HI absorption line allows us to distinguish a class of AGN/host interacting objects, to use as a lens for examining the host, the large-scale environment, radio-loudness and radio type, optical, x-ray, and IR luminosities, the level of dust obscuration, the narrow and broad emission lines, and other characteristics. However, the VLBI observations of Phase II will allow a truly detailed and comprehensive analysis of these properties, as well as their causes. 


\section{References}

Adelberger, K. L., \& Steidel, C. C. 2005, ApJ, 627, L1

Allevato, V., Finoguenov, A., Civano, F., et al. 2014, ApJ, 796, 4

Allison, J. R., Curran, S. J., Emonts, B. H. C., et al. 2012, MNRAS, 423, 2601

Alonso-Herrero, A. 2013, arXiv e-prints, arXiv:1302.2033

Alonso-Herrero, A., Rieke, G. H., Rieke, M. J., et al. 2006, ApJ, 650, 835

Ann, H. B., \& Thakur, P. 2005, ApJ, 620, 197

Antonucci, R. 1993, ARA\&A, 31, 473

Armus, L., Mazzarella, J. M., Evans, A. S., et al. 2009, PASP, 121, 559

Atkinson, J. W., Collett, J. L., Marconi, A., et al. 2005, MNRAS, 359, 504

Bahcall, J. N. 1969, ApJ, 158, L87

Baldwin, J. A., Phillips, M. M., \& Terlevich, R. 1981, PASP, 93, 5

Beckmann, V., Jean, P., Lubiński, P., Soldi, S., \& Terrier, R. 2011, A\&A, 531, A70

Bedregal, A. G., Colina, L., Alonso-Herrero, A., \& Arribas, S. 2009, ApJ, 698, 1852 
Bedregal, A. G., Colina, L., Azzollini, R., Arribas, S., \& Alonso-Herrero, A. 2011, in Astronomical Society of the Pacific Conference Series, Vol. 446, Galaxy Evolution: Infrared to Millimeter Wavelength Perspective, ed. W. Wang, J. Lu, Z. Luo, Z. Yang, H. Hua, \& Z. Chen, 83

Bennert, N., Canalizo, G., Jungwiert, B., et al. 2008, ApJ, 677, 846

Best, P. N., Kauffmann, G., Heckman, T. M., et al. 2005, MNRAS, 362, 25

Bolton, J. G., Clarke, M. E., Sandage, A., \& Véron, P. 1965, ApJ, 142, 1289

Bournaud, F., Elmegreen, B. G., Teyssier, R., Block, D. L., \& Puerari, I. 2010, MNRAS, 409, 1088

Bruzual, G., \& Charlot, S. 2003, MNRAS, 344, 1000

Cappelluti, N., Ajello, M., Burlon, D., et al. 2010, ApJ, 716, L209

Chandola, Y., Sirothia, S. K., Saikia, D. J., \& Gupta, N. 2012, Bulletin of the Astronomical Society of India, 40, 139

Chapman, S. C., Morris, S. L., \& Walker, G. A. H. 2000, MNRAS, 319, 666

Cohen, M., Walker, R. G., Carter, B., et al. 1999, AJ, 117, 1864

Coil, A. L., Hennawi, J. F., Newman, J. A., Cooper, M. C., \& Davis, M. 2007, ApJ, 654,115

Colina, L., García Vargas, M. L., Mas-Hesse, J. M., Alberdi, A., \& Krabbe, A. 1997, ApJ, 484, L41

Costagliola, F., Aalto, S., Sakamoto, K., et al. 2013, A\&A, 556, A66 
Couto, G. S., Storchi-Bergmann, T., Axon, D. J., et al. 2013, MNRAS, 435, 2982

Croft, S., van Breugel, W., de Vries, W., et al. 2006, ApJ, 647, 1040

Croom, S., Boyle, B., Shanks, T., et al. 2004, in Astronomical Society of the Pacific Conference Series, Vol. 311, AGN Physics with the Sloan Digital Sky Survey, ed. G. T. Richards \& P. B. Hall, 457

Croom, S. M., Boyle, B. J., Loaring, N. S., et al. 2002, MNRAS, 335, 459

Croom, S. M., Boyle, B. J., Shanks, T., et al. 2005, MNRAS, 356, 415

Cutri, R. M., Nelson, B. O., Francis, P. J., \& Smith, P. S. 2002, in Astronomical Society of the Pacific Conference Series, Vol. 284, IAU Colloq. 184: AGN Surveys, ed. R. F. Green, E. Y. Khachikian, \& D. B. Sanders, 127

Davies, R. I., Maciejewski, W., Hicks, E. K. S., et al. 2009, ApJ, 702, 114

de Vaucouleurs, G., de Vaucouleurs, A., Corwin, Jr., H. G., et al. 1991, Third Reference Catalogue of Bright Galaxies. Volume I: Explanations and references. Volume II: Data for galaxies between $0^{h}$ and $12^{h}$. Volume III: Data for galaxies between $12^{h}$ and $24^{h}$.

Díaz-Santos, T., Alonso-Herrero, A., Colina, L., et al. 2007a, in Revista Mexicana de Astronomia y Astrofisica Conference Series, Vol. 29, Revista Mexicana de Astronomia y Astrofisica Conference Series, ed. R. Guzmán, 92-94

Díaz-Santos, T., Alonso-Herrero, A., Colina, L., Ryder, S. D., \& Knapen, J. H. 2007b, ApJ, 661, 149

DiPompeo, M. A., Myers, A. D., Hickox, R. C., Geach, J. E., \& Hainline, K. N. 2014, MNRAS, 442, 3443 
Donley, J. L., Rieke, G. H., Rigby, J. R., \& Pérez-González, P. G. 2005, ApJ, 634, 169

Donoso, E., Li, C., Kauffmann, G., Best, P. N., \& Heckman, T. M. 2010, MNRAS, 407, 1078

Donoso, E., Yan, L., Stern, D., \& Assef, R. J. 2014, ApJ, 789, 44

Eisenstein, D. J., \& Hu, W. 1999, ApJ, 511, 5

Englmaier, P., \& Shlosman, I. 2000, ApJ, 528, 677

Espada, D., Matsushita, S., Peck, A. B., et al. 2012, ApJ, 756, L10

Evans, A. S., Vavilkin, T., Pizagno, J., et al. 2008, ApJ, 675, L69

Falder, J. T., Stevens, J. A., Jarvis, M. J., et al. 2010, MNRAS, 405, 347

—. 2011, ApJ, 735, 123

Farrah, D., Rowan-Robinson, M., Oliver, S., et al. 2001, MNRAS, 326, 1333

Farrah, D., Lonsdale, C. J., Borys, C., et al. 2006, ApJ, 641, L17

Fathi, K., Axon, D. J., Storchi-Bergmann, T., et al. 2011, ApJ, 736, 77

Ferrarese, L., \& Merritt, D. 2000, ApJ, 539, L9

Gebhardt, K., Bender, R., Bower, G., et al. 2000, ApJ, 539, L13

Gilli, R., Zamorani, G., Miyaji, T., et al. 2009, A\&A, 494, 33

Glikman, E., Gregg, M. D., Lacy, M., et al. 2004, ApJ, 607, 60

Greene, J. E. 2012, Nature Communications, 3, 1304 
Gregg, M. D., Lacy, M., White, R. L., et al. 2002, ApJ, 564, 133

Gupta, N., \& Saikia, D. J. 2006, MNRAS, 370, L80

Gupta, N., Salter, C. J., Saikia, D. J., Ghosh, T., \& Jeyakumar, S. 2006, MNRAS, 373,972

Gupta, N., Srianand, R., \& Saikia, D. J. 2005, MNRAS, 361, 451

Hickox, R. C., Myers, A. D., Brodwin, M., et al. 2011, ApJ, 731, 117

Ho, L. C. 2004, Coevolution of Black Holes and Galaxies, 496

Hopkins, P. F., Hernquist, L., Cox, T. J., \& Kereš, D. 2008, ApJS, 175, 356

Hopkins, P. F., Somerville, R. S., Hernquist, L., et al. 2006, ApJ, 652, 864

Horst, H., Duschl, W. J., Gandhi, P., \& Smette, A. 2009, A\&A, 495, 137

Inami, H., Armus, L., Surace, J. A., et al. 2010, AJ, 140, 63

Jogee, S., Shlosman, I., Laine, S., et al. 2003, in Astronomical Society of the Pacific Conference Series, Vol. 290, Active Galactic Nuclei: From Central Engine to Host Galaxy, ed. S. Collin, F. Combes, \& I. Shlosman, 437

Kanekar, N., \& Chengalur, J. N. 2008, MNRAS, 384, L6

Kennicutt, R. C., \& Evans, N. J. 2012, ARA\&A, 50, 531

Kormendy, J. 2001, in Revista Mexicana de Astronomia y Astrofisica Conference Series, Vol. 10, Revista Mexicana de Astronomia y Astrofisica Conference Series, ed. J. Cantó \& L. F. Rodríguez, 69-78

Lacy, M. 2000, ApJ, 536, L1 
Lacy, M., Gates, E. L., Ridgway, S. E., et al. 2002, AJ, 124, 3023

Lacy, M., Petric, A. O., Sajina, A., et al. 2007a, AJ, 133, 186

Lacy, M., Sajina, A., Petric, A. O., et al. 2007b, ApJ, 669, L61

Lacy, M., Storrie-Lombardi, L. J., Sajina, A., et al. 2004, ApJS, 154, 166

Lacy, M., Ridgway, S. E., Gates, E. L., et al. 2013, ApJS, 208, 24

Laine, S., Knapen, J. H., Perez-Ramirez, D., Doyon, R., \& Nadeau, D. 1999, MNRAS, 302, L33

Lauberts, A. 1982, ESO/Uppsala survey of the ESO(B) atlas

Longair, M. S., \& Seldner, M. 1979, MNRAS, 189, 433

Lonsdale, C. J., Smith, H. E., \& Lonsdale, C. J. 1995, ApJ, 438, 632

Lonsdale, C. J., Smith, H. E., Rowan-Robinson, M., et al. 2003, PASP, 115, 897

Loveday, J. 1996, MNRAS, 278, 1025

Lynden-Bell, D. 1969, Nature, 223, 690

Maciejewski, W. 2004a, MNRAS, 354, 883

-. 2004b, MNRAS, 354, 892

Magorrian, J., Tremaine, S., Richstone, D., et al. 1998, AJ, 115, 2285

Maia, M. A. G., Suzuki, J. A., da Costa, L. N., Willmer, C. N. A., \& Rite, C. 1996, A\&AS, 117, 487

Mandelbaum, R., Li, C., Kauffmann, G., \& White, S. D. M. 2009, MNRAS, 393, 377 
Martínez-Sansigre, A., Rawlings, S., Lacy, M., et al. 2005, Nature, 436, 666

Martini, P., Regan, M. W., Mulchaey, J. S., \& Pogge, R. W. 2003, ApJ, 589, 774

Mauduit, J.-C., Lacy, M., Farrah, D., et al. 2012, PASP, 124, 714

Modica, F., Vavilkin, T., Evans, A. S., et al. 2012, AJ, 143, 16

Morganti, R., Emonts, B., \& Oosterloo, T. 2009, A\&A, 496, L9

Morganti, R., Oosterloo, T. A., Emonts, B. H. C., van der Hulst, J. M., \& Tadhunter, C. N. 2003, ApJ, 593, L69

Morganti, R., Tadhunter, C. N., \& Oosterloo, T. A. 2005, A\&A, 444, L9

Mortlock, D. J., Warren, S. J., Venemans, B. P., et al. 2011, Nature, 474, 616

Myers, A. D., Brunner, R. J., Nichol, R. C., et al. 2007, ApJ, 658, 85

Myers, A. D., Brunner, R. J., Richards, G. T., et al. 2006, ApJ, 638, 622

Naim, A., Lahav, O., Buta, R. J., et al. 1995, MNRAS, 274, 1107

Netzer, H., \& Marziani, P. 2010, ApJ, 724, 318

Nilson, P. 1973, Nova Acta Regiae Soc. Sci. Upsaliensis Ser. V, 0

Oosterloo, T. 2004, in Astrophysics and Space Science Library, Vol. 312, High Velocity

Clouds, ed. H. van Woerden, B. P. Wakker, U. J. Schwarz, \& K. S. de Boer, 125

Oosterloo, T. A., \& Morganti, R. 2005, A\&A, 429, 469

Padmanabhan, N., White, M., Norberg, P., \& Porciani, C. 2009, MNRAS, 397, 1862

Papovich, C. 2008, ApJ, 676, 206 
Pastorini, G., Marconi, A., Capetti, A., et al. 2007, A\&A, 469, 405

Peterson, B. M. 2008, , 52, 240

Piqueras López, J., Colina, L., Arribas, S., \& Alonso-Herrero, A. 2013, A\&A, 553, A85

Piqueras López, J., Colina, L., Arribas, S., Alonso-Herrero, A., \& Bedregal, A. G. 2012, A\&A, 546, A64

Porciani, C., Magliocchetti, M., \& Norberg, P. 2004, MNRAS, 355, 1010

Prestage, R. M., \& Peacock, J. A. 1988, MNRAS, 230, 131

Reines, A. E. 2014, Nature, 513, 322

Reines, A. E., Greene, J. E., \& Geha, M. 2013, ApJ, 775, 116

Reines, A. E., Plotkin, R. M., Russell, T. D., et al. 2014, ApJ, 787, L30

Reines, A. E., Sivakoff, G. R., Johnson, K. E., \& Brogan, C. L. 2011, Nature, 470, 66

Reyes, R., Zakamska, N. L., Strauss, M. A., et al. 2008, AJ, 136, 2373

Ross, N. P., Shen, Y., Strauss, M. A., et al. 2009, ApJ, 697, 1634

Sandage, A., \& Tammann, G. A. 1995, VizieR Online Data Catalog, 7051, 0

Sanders, D. B., Mazzarella, J. M., Kim, D.-C., Surace, J. A., \& Soifer, B. T. 2003, AJ, 126, 1607

Sanders, D. B., \& Mirabel, I. F. 1996, ARA\&A, 34, 749

Sanders, D. B., Soifer, B. T., Elias, J. H., et al. 1988a, ApJ, 325, 74 
Sanders, D. B., Soifer, B. T., Elias, J. H., Neugebauer, G., \& Matthews, K. 1988b, ApJ, 328, L35

Sanders, D. B., Soifer, B. T., \& Scoville, N. Z. 1988c, Science, 239, 625

Sanders, R. H. 1998, MNRAS, 294, 35

Sargsyan, L., Weedman, D., Lebouteiller, V., et al. 2011, ApJ, 730, 19

Schawinski, K., Khochfar, S., Kaviraj, S., et al. 2006, Nature, 442, 888

Schinnerer, E., Böker, T., Emsellem, E., \& Lisenfeld, U. 2006, ApJ, 649, 181

Schmidt, M. 1963, Nature, 197, 1040

Schödel, R., Ott, T., Genzel, R., et al. 2002, Nature, 419, 694

Seldner, M., \& Peebles, P. J. E. 1978, ApJ, 225, 7

Serber, W., Bahcall, N., Ménard, B., \& Richards, G. 2006, ApJ, 643, 68

Seth, A. C., van den Bosch, R., Mieske, S., et al. 2014, Nature, 513, 398

Shen, Y., Strauss, M. A., Oguri, M., et al. 2007, AJ, 133, 2222

Shen, Y., Strauss, M. A., Ross, N. P., et al. 2009, ApJ, 697, 1656

Silk, J., \& Nusser, A. 2010, ApJ, 725, 556

Silk, J., \& Rees, M. J. 1998, A\&A, 331, L1

Soifer, B. T., Neugebauer, G., Matthews, K., et al. 2001, AJ, 122, 1213

Spoon, H. W. W., Keane, J. V., Tielens, A. G. G. M., Lutz, D., \& Moorwood, A. F. M. 2001, A\&A, 365, L353 
Springel, V., White, S. D. M., Jenkins, A., et al. 2005, Nature, 435, 629

Stefanon, M., \& Marchesini, D. 2013, MNRAS, 429, 881

Stern, D., Eisenhardt, P., Gorjian, V., et al. 2005, ApJ, 631, 163

Stierwalt, S., Armus, L., Surace, J. A., et al. 2013, ApJS, 206, 1

Stierwalt, S., Armus, L., Charmandaris, V., et al. 2014, ApJ, 790, 124

Storchi-Bergmann, T., Dors, Jr., O. L., Riffel, R. A., et al. 2007, ApJ, 670, 959

Surace, J. A., Sanders, D. B., Vacca, W. D., Veilleux, S., \& Mazzarella, J. M. 1998, ApJ, 492, 116

Teng, S. H., Veilleux, S., \& Baker, A. J. 2013, ApJ, 765, 95

Thacker, R. J., Scannapieco, E., Couchman, H. M. P., \& Richardson, M. 2009, ApJ, 693,552

Thakur, P., Ann, H. B., \& Jiang, I.-G. 2009, ApJ, 693, 586

Treister, E., Natarajan, P., Sanders, D. B., et al. 2010, Science, 328, 600

Treister, E., Schawinski, K., Urry, C. M., \& Simmons, B. D. 2012, ApJ, 758, L39

Treister, E., \& Urry, C. M. 2006, ApJ, 652, L79

Treister, E., Urry, C. M., Chatzichristou, E., et al. 2004, ApJ, 616, 123

Treister, E., Cardamone, C. N., Schawinski, K., et al. 2009, ApJ, 706, 535

Turner, J. L., \& Hurt, R. L. 1992, ApJ, 384, 72

Ueda, Y., Eguchi, S., Terashima, Y., et al. 2007, ApJ, 664, L79 
Urrutia, T., Lacy, M., \& Becker, R. H. 2008, ApJ, 674, 80

Urry, C. M., \& Padovani, P. 1995, PASP, 107, 803

Véron-Cetty, M.-P., \& Véron, P. 2006, A\&A, 455, 773

Villforth, C., Hamann, F., Rosario, D. J., et al. 2014, MNRAS, 439, 3342

Wake, D. A., Collins, C. A., Nichol, R. C., Jones, L. R., \& Burke, D. J. 2005, ApJ, 627,186

Wake, D. A., Miller, C. J., Di Matteo, T., et al. 2004, ApJ, 610, L85

Walterbos, R. A. M., \& Graeve, R. 1985, A\&A, 150, L1

Wild, V., Kauffmann, G., White, S., et al. 2008, MNRAS, 388, 227

Wold, M., Lacy, M., Lilje, P. B., \& Serjeant, S. 2000, MNRAS, 316, 267

-. 2001, MNRAS, 323, 231

Wylezalek, D., Galametz, A., Stern, D., et al. 2013, ApJ, 769, 79

Yee, H. K. C., \& Green, R. F. 1984, ApJ, 280, 79

Yee, H. K. C., \& López-Cruz, O. 1999, AJ, 117, 1985

Zakamska, N. L., Strauss, M. A., Krolik, J. H., et al. 2003, AJ, 126, 2125 